\title{
Study on Flavonoid and Bioactivity Features of the Pericarp of Citri Reticulatae 'Chachi' During Storage
}

Shejian Liang

South China Agricultural University College of Life Sciences

Zhijia Wen

South China Agricultural University College of Life Sciences

Tiexin Tang

Zhaoqing Medical College

Yufang Liu

South China Agricultural University College of Life Sciences

Fengliang Dang

South China Agricultural University College of Life Sciences

Tianxiao Xie

South China Agricultural University College of Life Sciences

Hong Wu ( $\nabla$ wh@scau.edu.cn)

South China Agricultural University College of Life Sciences https://orcid.org/0000-0001-9142-499X

\section{Research}

Keywords: pericarp of Citri Reticulatae 'Chachi,' aging period, flavonoids, UPLC-MS/MS, antioxidation, SARS-CoV-2

Posted Date: August 10th, 2021

DOI: https://doi.org/10.21203/rs.3.rs-753313/v1

License: (c) (i) This work is licensed under a Creative Commons Attribution 4.0 International License. Read Full License

Version of Record: A version of this preprint was published at Arabian Journal of Chemistry on December 1st, 2021. See the published version at https://doi.org/10.1016/j.arabjc.2021.103653. 


\section{Abstract}

Background: The mature pericarp of Citri Reticulatae 'Chachi' (PCRC) is one of the six traditional Chinese medicinal materials that should be used after long storage, and it was regarded that the longer the medicine was stored, the better. However, the aging mechanism of the medicine is not clear.

Methods: In order to further investigate the effect of aging on the main active flavonoids of PCRC, ultra-performance liquid chromatography coupled with triple quadrupole mass spectrometry (UPLC-MS/MS) and metabolomics analysis were used to analyze the flavonoids of PCRC stored for different periods.

Results: In the results, 219 flavonoids were detected. 5,7,3',4',5'-pentamethoxy dihydroflavone and 2'-hydroxy-3,4,5,3'4',6'-hexamethoxychalcone were found from PCRC for the first time. According to the clustering analysis of metabolites, aging times of 0 , 1 , and 2 were clustered into one group, and aging times of 3 , 4 , and 29 were clustered into the other group. Quantitative analysis showed that the former group contained a greater amount of 4 flavonoids than the latter group, while the latter group contained a greater amount of 15 polymethoxyflavonoids. The newly harvested PCRC was compared with the other 5 groups of PCRC (stored for 1, 2, 3, 4, and 29 years). Eight flavonoids, tectochrysin, apigenin, 2'-hydroxyisoflavone, luteolin, 6-hydroxyluteolin, gallocatechin, quercetin -0acetylhexoside and apigenin -7-O-[ $\beta$-D-glucuronide $(1 \rightarrow 2)$-O-B-D-glucuronide], were used as marker components to discriminate newly harvested PCRC and aging PCRC. In addition, the antioxidant potency composite index (APC) indicated that the PCRC stored for three or four years had stronger antioxidant activity than the PCRC stored for other periods. By means of molecular docking, it was reviewed that the amount of antiviral components against SARS-CoV-2 in freshly harvested PCRC was significantly higher than that in aging PCRC. The results in this study supplied scientific data for quality control, evaluation, and rational utilization of PCRC and basic information for further analysis of the metabolic regulation of the active components of the PCRC.

\section{Introduction}

The traditional Chinese medicinal material, 'Chenpi' (Citri Reticulatae Pericarpium), is the dry mature pericarp of Citrus reticulata Blanco and its cultivars. According to the cultivation place and cultivar, Citri Reticulatae Pericarpium was divided into two types, 'Chenpi' and 'Guang Chenpi' [1]. 'Guang Chenpi' refers to the Citri Reticulatae Pericarpium cultivated in Xinhui, Guangdong, China, which is the dry, mature pericarp of the cultivar Citrus reticulata Blanco, Citrus reticulata 'Chachi.' The dry, mature pericarp of Citrus reticulata 'Chachi' (PCRC) is of higher quality. Modern phytochemical studies have shown that the chemical constituents of PCRC are flavonoids, volatile oils, polysaccharides, and alkaloids [2]. The main active components are flavonoids, including flavonoid glycosides and polymethoxyflavonoids [3]. According to the Chinese Pharmacopoeia, PCRC regulates qi flow to strengthen the spleen and dry dampness to reduce phlegm. It is mainly used to treat abdominal distension, lack of appetite, vomiting, and diarrhea, and coughing with phlegm [1]. Modern pharmacological studies also show that it has antioxidative, antibacterial, antiviral, anti-inflammatory, antitumor, and hypolipidemic effects [4-9]. It has a long history that PCRC was used as medicine. PCRC was recorded in a pharmaceutical monograph written approximately 2000 years ago in the Eastern Han Dynasty, "Shennong's classic of materia medica" [10]. Currently, the number of preparations employing PCRC recorded in the Chinese Pharmacopoeia (2020 Edition) is as high as 176 [1]. In addition, PCRC is also the most popular food seasoning or ingredient in China. Among them, the annual market sales of PCRC produced from Xinhui reached 10 billion RMB, ranking first for three consecutive years in influence ranking of agricultural products on development forum for the regional agricultural brand of China .

In traditional Chinese medicine, there is the theory of "aging medicine." Aging medicine refers to the medicinal material stored and maintained by specific methods and then used after aging. The process of storage, maintenance, and aging is the process of changing the properties and efficacy of the medicinal material, making it more suitable to the clinical needs of traditional Chinese medicine [11]. As early as 1500 years ago, in the Southern Dynasties.Liang Dynasty, Hongjing Tao recorded Citri Reticulatae Pericarpium as one of the six aging traditional Chinese medicinal materials, which should be used after aging [12]. Since then, in the Tang, Song, Yuan, Ming, and Qing Dynasties, there have been records that the longer the Citri Reticulatae Pericarpium was stored, the better [12-17]. Looking up all the ancient books recording traditional Chinese medicinal materials, the reasons for long storage treatment of aging medicine were not discussed [18]. In recent years, with the help of modern instrumental analysis, researchers have rapidly promoted the development of research on the effect of aging on the active components of Citri Reticulatae Pericarpium. Wang et al.[19] studied the effect of aging on the component accumulation and biological activity of Citri Reticulatae Pericarpium. The results showed that the content of combined polyphenols and flavonoids increased significantly during the aging process, and the antioxidant activity increased. By means of HPLC, Liu et al [20] showed that the contents of three flavonoids, hesperidin, nobiletin, and hesperetin, significantly increased as the storage time was prolonged. Zheng et al.[21] quantitatively studied the changes in five flavonoids, hesperidin, nobiletin, 3,5,6,7,8,3',4'-heptamethoxyflavone, hesperetin, and 5-hydroxy-6,7,8,3',4'-pentamethoxyflavone, in ten batches of PCRC with different storage periods, and the results showed that the contents of five flavonoids tended to increase as storage time increased. Fu et al.[22] determined Citri Reticulatae Pericarpium stored for 36 months by HPLC-dual wavelength detection and found that the content of five flavoids of sinensetin, 4,5,7,8-tetramethoxyflavone, nobiletin, hesperetin, and 5-0-demethylnobiletin increased, while the content of hesperidin decreased. In the above results, the characteristics of specific components in the aging process of PCRC were not consistent with each other and were even contradictory. The reason for the deviation of those results was hard to analyze. However, based on metabonomics, a study indicated that up to 92 flavonoids were determined in the pericarp of freshly harvested Citrus reticulata 'Chachi' [23]. Currently, most published works have determined only $3 \sim 5$ flavonoids to study the change in flavonoids in PCRC during aging. It was hard to show the whole pattern. In this study, to comprehensively evaluate the change of flavonoids in PCRC during aging, UPLC-MS/MS and widely targeted metabolomics analyses were employed to fully investigate the quantity and composition of flavonoids, and the antioxidant potency composite index (APC) was determined to compare the activity. Moreover, the novel coronavirus found at the end of 2019 was named 2019 novel coronavirus or "2019-nCoV" by the World Health Organization (WHO) on January 12, 2020 [24]. As of June 12, 2021, Beijing time, more than 176 million people had been infected with COVID-19, and more than 3.8 million people had died all over the world. Unfortunately, there is currently no specific drug for COVID-19 in the world. Wu reported that flavonoids such as neohesperidin, hesperidin, baicalin, kaempferol 3-0-rutinoside, and rutin from different sources, with antiviral, antibacterial, and anti-inflammatory activities, could effectively interact with some targets of SARS-CoV-2 [24]. Therefore, to study the possible effect of the abundant flavonoids in PCRC on the prevention and treatment of COVID-19, further study by means of molecular docking was used to analyze the binding affinity of some important flavonoid glycosides and polymethoxyflavones at target proteins of SARS-CoV-2. The results in this study showed that the aging process promoted the accumulation of 
important pharmacologically active compounds, polymethoxyflavones, and supplied evidence of active components to the statement of "the longer the medicine was stored the better" for PCRC. The contents of narirutin, neohesperidin, and hesperidin with higher binding affinity with target proteins in SARS-

CoV-2 were most abundant in freshly harvested PCRC. The results supplied scientific data for the quality control, evaluation, and rational utilization of PCRC.

\section{Materials And Methods}

\subsection{Plant materials}

The fruits of Citrus reticulata 'Chachi' were harvested in November 1990, 2015, 2016, 2017, 2018, and 2019 from Liangmei Farm, Fumei village, Shuangshui town, Xinhui District, Jiangmen City, Guangdong Province. Gatherer was Shu-shen Zhang. All samples were identified to be genuine by associate Professor Rong-jing Zhang. After harvest, stains on the surface of the fruits were washed off with clean water. Pericarps were peeled off the fruits, dried under sunlight, and put in sealed bags for storage. The PCRC was stored in a cool and dry place for aging. They were removed and dried under sunlight several times a year. Sample information is listed in Table 1.

Table 1

PCRC samples information

\begin{tabular}{|lllll|}
\hline No. & $\begin{array}{l}\text { Harvest and preparation } \\
\text { time }\end{array}$ & $\begin{array}{l}\text { Aging period } \\
\text { (year) }\end{array}$ & Common name & Origin \\
\hline C0 & Nov, 2019 & 0 & $\begin{array}{l}\text { Freshly harvested } \\
\text { pericarp }\end{array}$ & $\begin{array}{l}\text { Fumei, village, Shuangshui town, Xinhui district, Jiangmen, } \\
\text { Guangdong }\end{array}$ \\
\hline C1 & Nov, 2018 & 1 & 1 year pericarp \\
\hline C2 & Nov, 2017 & 2 & 2 year pericarp \\
\hline C3 & Nov, 2016 & 3 & 3 year pericarp \\
\hline C4 & Nov, 2015 & 4 & 4 year pericarp \\
\hline C29 & Nov, 1990 & 29 & 29 year pericarp \\
\hline
\end{tabular}

\subsection{Sample preparation}

\subsubsection{Sample preparation and extraction for flavonoid metabolomic analysis}

The freeze-dried samples were crushed using a mixer mill (MM 400, Retsch) for $1.5 \mathrm{~min}$ at $30 \mathrm{~Hz}$. One hundred milligrams of powder from each sample was weighed and extracted with $1.0 \mathrm{~mL} 70 \%$ aqueous methanol. The resulting mixtures were stored at $4^{\circ} \mathrm{C}$ overnight and vortexed three times to ensure complete extraction. Following centrifugation at $10,000 \mathrm{~g}$ for $10 \mathrm{~min}$, the extracts were absorbed and filtered (SCAA-104, $0.22 \mu \mathrm{m}$ pore size) before UPLC-MS/MS analysis.

\subsubsection{Quality control (QC) samples}

QC samples were prepared by blending the extracts of samples in all groups with equal amounts. Four replicate QC samples were prepared separately, named mix01 to mix04. QC samples were determined with the same method as well as the samples. In instrumental analysis, one QC sample was determined after every three samples to evaluate the repeatability of the whole analysis process.

\subsection{Methods}

\subsubsection{Determination of total flavonoid content and total antioxidant activity}

Three pieces of PCRC were randomly removed, powdered by a pulverizer, and sieved through a 40 mesh sieve. PCRC powder accurately weighing $0.5 \mathrm{~g}$ was transferred into a conical flask, followed by $32 \mathrm{~mL}$ of $70 \%$ aqueous methanol solution. The mixture was treated with an ultrasonic cleaner (ultrasonic power $100 \mathrm{~W}$, water temperature $60^{\circ} \mathrm{C}$ ) for 1 hour. Then, it was centrifuged at $6000 \mathrm{rpm}$ for $10 \mathrm{~min}$. Supernatant liquid was collected. The extraction was repeated three times. The supernatant liquid of three extractions was combined and diluted to $100 \mathrm{~mL}$ in a volumetric flask to give the sample solution. Three replicate sample solutions were prepared separately from every PCRC sample. Determination of total flavonoid content adopted from the method of Meyers et al [25]. was slightly adapted. Determination of total antioxidant activity employed a detection kit from Beyotime Biotechnology Co., Ltd. and followed the FRAP and ABTS procedures. The DPPH method referred to the method of Kong et al [26]. with a small adaption.

\subsubsection{HPLC Conditions}

The sample extracts were analyzed using an LC-ESI-MS/MS system (HPLC, Shim-pack UFLC SHIMADZU CBM30A system, www.shimadzu.com.cn/; MS, Applied Biosystems 6500 Q TRAP,www.appliedbiosystems. com.cn/). The analytical conditions were as follows, HPLC: column, Waters ACQUITY UPLC HSS T3 C18 $(1.8 \mu \mathrm{m}, 2.1 \mathrm{~mm} \star 100 \mathrm{~mm})$; solvent system, water (0.04\% acetic acid): acetonitrile $(0.04 \%$ acetic acid); gradient program,100:0 V/V at $0 \mathrm{~min}, 5: 95 \mathrm{~V} / \mathrm{V}$ at $11.0 \mathrm{~min}, 5: 95 \mathrm{~V} / \mathrm{V}$ at $12.0 \mathrm{~min}, 95: 5 \mathrm{~V} / \mathrm{V}$ at $12.1 \mathrm{~min}, 95: 5 \mathrm{~V} / \mathrm{V}$ at $15.0 \mathrm{~min}$; flow rate, $0.40 \mathrm{~mL} / \mathrm{min}$; temperature, $40^{\circ} \mathrm{C}$; injection volume: $2 \mu \mathrm{L}$. The effluent was alternatively connected to an ESI-triple quadrupole-linear ion trap (Q TRAP)-MS.

\subsubsection{ESI-Q TRAP-MS/MS}


LIT and triple quadrupole (QQQ) scans were acquired on a triple quadrupole-linear ion trap mass spectrometer (Q TRAP), API 6500 Q TRAP LC/MS/MS System, equipped with an ESI Turbo Ion-Spray interface, operating in positive ion mode and controlled by Analyst 1.6.3 software (AB Sciex). The ESI source operation parameters were as follows: ion source, turbo spray; source temperature $500^{\circ} \mathrm{C}$; ion spray voltage (IS) $5500 \mathrm{~V}$; ion source gas I (GSI), gas II(GSII), curtain gas (CUR) were set at 55, 60, and 25.0 psi, respectively; the collision gas (CAD) was high. Instrument tuning and mass calibration were performed with 10 and $100 \mu \mathrm{mol} / \mathrm{L}$ polypropylene glycol solutions in QQQ and LIT modes, respectively. QQQ scans were acquired as MRM experiments with collision gas (nitrogen) set to $5 \mathrm{psi}$. DP and CE for individual MRM transitions were performed with further DP and CE optimization. A specific set of MRM transitions was monitored for each period according to the metabolites eluted within this period.

\subsubsection{Molecular docking}

The protein structures of the spike (PDB ID: 6 VSB, chains A, B, C), 3CLpro (PDB ID: 6 LU7, chain A), PLpro (PDB ID: 4OVZ, chain A), and RdRp (PDB ID: 6 NUS, chain A), four potential antiviral targeting proteins for SARS-CoV-2, were downloaded from the Protein Data Bank (www.rcsb.org). Three-dimensional molecular structures of flavonoids and positive control drugs were downloaded from the compound database of PubChem

(https://pubchem.ncbi.nlm.nih.gov/). Lopinavir, ritonavir, ribavirin, chloroquine, arbidol, and favipiravir were used as positive controls because they were recommended in the COVID-19 Diagnosis and Treatment Plan (trial version 8) [27].

Three-dimensional molecular structures of flavonoids and positive control drugs were ligands. The protein structures of Spike, 3CLpro, PLpro, and RdRp were used for binding. Molecular docking experiments employed Auto Dock Vina software. A grid box (126 $\AA \times 126 \AA \times 126 \AA)$ centered at $226.49,226.728,221.894 \AA$ was used for the spike. A grid box $(60 \AA \AA \times 60 \AA \times 60 \AA)$ centered at $(-28.059,9.486,61.528) \AA$ was used for $3 C L$ pro. A grid box $(60 \AA \times 60 \AA \times 60 \AA)$ centered at $(-13.953,51.391,-31.750) \AA$ was used for PLpro. A grid box $(126 \AA \times 126 \AA \times 126 \AA)$ centered at $(152.179,167.664,166.985) \AA$ was used for the RdRp.

\subsection{Data analysis}

\subsubsection{PCA}

Unsupervised PCA (principal component analysis) was performed by the statistics function prcomp within R (www.r-project.org). The data were unit variance scaled before unsupervised PCA.

\subsubsection{Hierarchical Cluster Analysis and Pearson Correlation Coefficients}

The HCA (hierarchical cluster analysis) results of samples and metabolites are presented as heatmaps with dendrograms, while Pearson correlation coefficients (PCCs) between samples were calculated by the cor function in R and presented as only heatmaps. Both HCA and PCC were carried out by R package heatmap. For HCA, normalized signal intensities of metabolites (unit variance scaling) are visualized as a color spectrum.

\subsubsection{Differential metabolites selected}

Significantly regulated metabolites between groups were determined by VIP $\geq 1$ and absolute Log2FC (fold change) $\geq 1$. VIP values were extracted from the OPLS-DA results, which also contained score plots and permutation plots, and were generated using the R package MetaboAnalystR. The data were logtransformed (log2) and mean-centered before OPLS-DA. To avoid overfitting, a permutation test (200 permutations) was performed.

\subsubsection{KEGG annotation and enrichment analysis}

Identified metabolites were annotated using the Kyoto Encyclopedia of Genes and Genomes (KEGG) database, and annotated metabolites were then mapped to the KEGG pathway database. Pathways with significantly regulated metabolites mapped to were then fed into MSEA (metabolite set enrichment analysis), and their significance was determined by hypergeometric test $p$-values.

\section{Results}

\subsection{Determination of total flavonoid content}

Total flavonoid contents of PCRC from six groups with different aging periods were determined by spectrophotometry. The results showed that with increasing aging time, the total flavonoid contents of PCRC first decreased, then increased, and then decreased again (Fig. 2). Three groups with shorter aging periods $(\mathrm{C} 0, \mathrm{C} 1, \mathrm{C} 2)$ had a lower total flavonoid content than three groups with longer aging periods (C3, C4, C29). The total flavonoid content of the C3 group was the highest, which was $0.83 \%$. This result indicated that different aging periods had different impacts on the flavonoids of PCRC.

\subsection{Metabolism features of flavonoids}

\subsubsection{Qualitative and quantitative analysis of flavonoid metabolites}

To evaluate the effect of aging on flavonoids of PCRC, widely targeted metabolomics analysis was employed for the six groups of PCRC. Figures 3a, 3b show the total ion current (TIC) chromatogram of quality control samples. Figures 3c, 3d show multipeak chromatograms of metabolites using multiple reaction monitoring (MRM). Based on the MetWare database, the metabolites of samples were determined qualitatively and quantitatively by UPLC-MS/MS. Under MRM mode, multipeak chromatograms of metabolites show detectable components. Every color indicated one metablite. Characteristic ions were screened by triple quadrupole, and their signal strength was detected on the detector. Data files were opened by MultiaQuant software to integrate peaks and make calibrations. The peak area indicates the relative content of the component. In this study, 219 flavonoid metabolites were detected, including 82 flavones, 56 
flavonols, 26 flavonoid C-glycosides, 19 dihydroflavones, 7 isoflavones, 7 tannins, 5 flavanols, 5 dihydroflavonols, 4 anthocyanins, 3 chalcones, 2 other flavonoids, 2 proanthocyanidins, and 1 dihydroisoflavone. Among them, 5,7,3',4',5'-pentamethoxy-dihydroflavones and 2'-hydroxy-3,4,5,3',4',6'hexamethoxychalcone. were detected in PCRC for the first time. The metabolites detected are listed in Additional file 1: Table S1, including precursor, integration value, and corresponding metabolite name.

\subsubsection{Multivariate analysis of the metabolites}

The ion current strength data of the flavonoid metabolites were used to establish a cluster heat map, and the samples were analyzed by principal component analysis (PCA). A cluster heat map showed that three replicate samples from the same groups clustered and indicated that the data were reliable (Fig. $4 \mathrm{a}$ ). In addition, clustering results of samples from different aging groups showed that six groups of different aging periods first clustered into two classes. C0, $\mathrm{C}$, and $\mathrm{C} 2$ clustered into one class, and C3, C4, and C29 clustered into the other. According to the length of the aging, they were further divided into four classes: $\mathrm{C} 0$ was one class by itself, $\mathrm{C} 1$ and $\mathrm{C} 2$ were clustered into a class, C3 was one class by itself, and C4 and C29 were clustered into a class. This result indicated that flavonoid metabolites of PCRC varied with the length of the aging, and flavonoid metabolites of PCRC were more similar as their lengths of the aging were more closed. In Fig. 4b, PC1 and PC2 explained $71.28 \%$ of the total difference, and three replicate samples from the same groups were combined. C1 was close to C2. C4 was close to C29. The results were consistent with the cluster heat map.

\subsection{Screening and analysis of the differential metabolites between freshly harvested PCRC and aging PCRC}

By means of clustering analysis and PCA of the 6 groups of PCRC samples, it was found that the flavonoid metabolites significantly varied over the aging period. Comparing the ion strength of flavonoids in PCRC samples with different aging periods, differential metabolites were screened out. The results showed

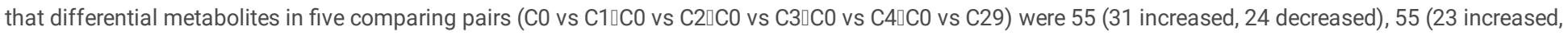
32 decreased), 58 (45 increased, 13 decreased), 51 (30 increased, 21 decreased), 49 (27 increased, 22 decreased) (Fig. 5), separately. Overall, the content of $45.2 \%$ metabolites (99 components) changed significantly. This result indicated that the aging process significantly affected the content of flavonoid metabolites. To identify the differential metabolites from the flavonoid metabolites between freshly harvested PCRC and the other five groups of aging PCRC (C1, C2, C3, C4, C29), a Venn diagram was employed (Fig. 6). There were 21 common flavonoid metabolites among the five pairs of PCRC groups: C0 vs C1, C0 vs C2, C0 vs C3, C0 vs C4, and C0 vs C29(Table 2). Seven flavonoids (tectochrysin, apigenin, 2'-hydroxyisoflavone, luteolin, 6-hydroxyluteolin, gallocatechin, quercetin-O-acetylhexoside) were detected in five groups of aging PCRC samples and were not detected in freshly harvested PCRC samples. Apigenin-7-O-[ $\beta$-Dglucuronide $(1 \rightarrow 2)-0-\beta-D$-glucuronide] was only detected in freshly harvested PCRC samples.

\subsection{Analysis of contents change of main flavonoids}

Flavonoids are a kind of main active component in PCRC, including flavonoid glycosides and polymethoxyflavones. Fifteen polymethoxyflavones were screened out from 219 detected metabolites of flavonoids. They were tangerine, nobiletin, 5-hydroxy-6,7,8,3',4'-pentamethoxyflavone (demethylnobiletin), 3,5,6,7,8,3', 4'-heptamethoxyflavone, 5-hydroxy-6,7,3',4'-tetramethoxyflavone, 5,7,8,4'-tetramethoxyflavone, monohydroxy-hexamethoxyflavone, 7-hydroxy-3,5,6,8tetramethoxyflavone, sinensetin (5,6,7,3',4'-pentamethoxyflavone), 5,6,7,8,3',4'-hexamethoxyflavone, isosinensetin (3',4', 5,7,8-pentamethoxyflavone), skullcapflavone II(5,6'- dihydroxy-6,7,8,2'-tetramethoxyflavone), natsudaidain(3-hydroxy-5,6,7,8,3', 4'-hexamethoxyflavone), 5,7,3',4',5'pentamethoxydihydroflavone,and 2'-hydroxy-3,4,5,3'4',6'-hexamethoxychalcone. 5,7,3',4',5'-pentamethoxydihydroflavone was first detected in PCRC. To make the change of the contents of flavonoid glycosides and polymethoxyflavones in PCRCs of different aging periods more obvious, the relative content of flavonoid glycosides (Fig. 7) and polymethoxyflavones (Fig. 8,9) were compared in the form of histograms.

The flavonoid glycosides with higher relative contents in PCRC were hesperidin, neohesperidin, naringin, and narirutin. The polymethoxyflavones with higher relative contents in PCRC were tangerine and nobiletin. By comparison, it was found that as the aging period extended, four flavonoid glycosides, hesperidin, neohesperidin, naringin, and narirutin, decreased, while polymethoxyflavones increased.

As shown in Fig. 7, as the aging period was extended, the neohesperidin content decreased. The neohesperidin content of the C3 group was not significant; however, the trend generally decreased. The hesperidin content also decreased as the aging period extended. After three years, the change in hesperidin content was not significant; however, it was still decreasing. Narirutin content decreased at first and then increased in the first two years of storage, slowly decreased after two years, and did not change significantly after four years. The change in naringin content was in accordance with the change in narirutin content. Generally, in the aging process, the changes in the contents of the four flavonoid glycosides slightly fluctuated but eventually significantly decreased. Flavonoid glycosides in PCRC decreased as the aging period extended.

In Fig. 8, the contents of tangerine and nobiletin slightly fluctuated; however, the change was not significant. Generally, their contents in the groups with shorter aging periods $(C 0, C 1, C 2)$ were lower than those in the groups with longer aging periods ( $C 3, C 4, C 29)$, and the change was not significant among the $C 3$, $C 4$, C29 groups. The content of 5-hydroxy-6,7,8,3',4'-pentamethoxyflavone (demethylnobiletin) fluctuated greatly, and the content in the C0, C3, and C29 groups did not change significantly and was higher than that in the other groups. The content of 3,5,6,7,8,3', '-heptamethoxyflavone did not change significantly from group $\mathrm{C} 0$ to group $\mathrm{C} 1$, increased gradually from group $\mathrm{C} 1$ to group $\mathrm{C} 4$, and decreased slightly in groups $\mathrm{C} 4$ and C29. The content of 5-hydroxy-6,7,3',4'tetramethoxyflavone fluctuated and was highest in group C3 and then decreased. The content of 5,7,8,4'-tetramethoxyflavone decreased from group C0 to group C1, increased gradually from group C1 to group C3, and slightly decreased from C3 to group C29. The contents of monohydroxy-hexamethoxyflavone and 7-hydroxy-3,5,6,8-tetramethoxyflavone had similar changing trends, which gradually decreased from group C0 to group C2, sharply increased from group C2 to group C3, and fluctuated from group C3 to group C29 with relatively slight changes.

In Fig. 9, the content of sinensetin (5,6,7,3',4'-pentamethoxyflavone) first decreased and then increased to its highest level from group C0 to C3 and did not change significantly thereafter. The content of 5,6,7,8,3',4'-hexamethoxyflavone increased to its highest level from group $\mathrm{C} 0$ to group $\mathrm{C} 4$ and slightly decreased 
from group C4 to group C29. The content of natsudaidain (3-hydroxy-5,6,7,8,3',4'-hexamethoxyflavone) slightly fluctuated and was lower in the groups with shorter aging periods $(\mathrm{C} 0, \mathrm{C} 1, \mathrm{C} 2)$ than in the groups with longer aging periods (C3, C4, C29). The content of isosinensetin ( $3^{\prime}, 44^{\prime}, 5,7,8-$-pentamethoxyflavone) was nearly unchanged from group $\mathrm{C} 0$ to group $\mathrm{C} 2$, increased to its highest level from group $\mathrm{C} 2$ to group $\mathrm{C} 3$, and gradually decreased thereafter. The content of skullcapflavone II(5,6'- dihydroxy-6,7,8,2'-tetramethoxyflavone) had a change of fluctuation, which gradually decreased from groups $\mathrm{C} 0$ to $\mathrm{C} 2$, increased from group C2 to group C3, and fluctuated from group C3 to group C29. The content of 5,7,3', $4^{\prime}, 5^{\prime}$-pentamethoxydihydroflavone remained stable from group C0 to group C2, then increased, and obtained the highest content in group C29. The content of 2'-hydroxy-3,4,5,3'4',6'-hexamethoxychalcone steadily increased from group C0 to group C29.

Table 221 common flavonoid metabolites in C0_vs_C1, C0_vs_C2, C0_vs_C3, C0_vs_C4, and C0_vs_C2

\begin{tabular}{|c|c|c|c|c|c|c|}
\hline \multirow[t]{2}{*}{ Components } & \multicolumn{6}{|l|}{ Relative content } \\
\hline & $\mathrm{CO}$ & $\mathrm{C} 1$ & $\mathrm{C} 2$ & C3 & C4 & C \\
\hline Methyl gallate & $19785 \pm 1931 f$ & $106395 \pm 15635 e$ & $157937 \pm 11140 d$ & $275190 \pm 19796 c$ & $463257 \pm 21037 b$ & 6 \\
\hline Tectochrysin & Oc & $21822 \pm 888 a$ & $20924 \pm 1532 a b$ & $22378 \pm 898 a$ & $18803 \pm 1959 b$ & 1 \\
\hline Apigenin & $0 d$ & $69684 \pm 3765 c$ & $194160 \pm 23714 b$ & $176273 \pm 13523 b$ & $234647 \pm 15452 a$ & 1 \\
\hline 2'-Hydroxyisoflavone & Od & $5850 \pm 1480 c$ & $858 \pm 3963 b c$ & $11922 \pm 3714 b$ & $40597 \pm 3258 a$ & 1 \\
\hline Luteolin & Oe & $99007 \pm 2736 d$ & $183020 \pm 31610 \mathrm{c}$ & $399967 \pm 49360 a$ & $321533 \pm 39569 b$ & 1 \\
\hline Isosakuranetin & $126767 \pm 10722 f$ & $888373 \pm 49580 e$ & $1784533 \pm 102701 a$ & $1090167 \pm 75982 d$ & $1629566 \pm 57415 b$ & 1 \\
\hline Eriodictyol & $76828 \pm 2182 e$ & $533217 \pm 7574 d$ & $990117 \pm 103771 b$ & $1122450 \pm 108947 a$ & $851797 \pm 17286 c$ & 5 \\
\hline 6-Hydroxyluteolin & $\mathrm{Oe}$ & $36007 \pm 7907 d$ & $80031 \pm 11845 c$ & $109570 \pm 7182 b$ & $110593 \pm 6913 b$ & 4 \\
\hline Homoeriodictyol & $1007713 \pm 177275 e$ & $59225333 \pm 4433949 c$ & $136000000 \pm 10570000 a$ & $59222667 \pm 6087200 c$ & $88361667 \pm 2979724 b$ & 4 \\
\hline Hesperetin & $417317 \pm 52441 d$ & $26183667 \pm 965291 \mathrm{c}$ & $67591333 \pm 5854918 a$ & $29585667 \pm 3944180 c$ & $44605333 \pm 838270 \mathrm{~b}$ & 2 \\
\hline Gallocatechin & Od & $4142 \pm 7174 d$ & $40853 \pm 13747 b$ & $91122 \pm 8330 a$ & $24953 \pm 3451 c$ & 1 \\
\hline Galloylgallic acid & $1147 \pm 1020 d$ & Od & Od & $12176 \pm 2365 c$ & $29879 \pm 3464 b$ & 5 \\
\hline $\begin{array}{l}5,6,7,8,3 \text { ', } 4^{\prime}- \\
\text { Hexamethoxyflavanone }\end{array}$ & $588443 \pm 30549 f$ & $3761167 \pm 134203 e$ & $5750100 \pm 163503 d$ & $10055433 \pm 231343 b$ & $10927667 \pm 297248 a$ & 8 \\
\hline $\begin{array}{l}\text { 5,4'-Dihydroxy-6,7- } \\
\text { dimethoxyflavone-8-C- } \\
\beta \text {-D-glucoside }\end{array}$ & $67129 \pm 10490 d$ & $239023 \pm 95537 \mathrm{c}$ & $340777 \pm 109329 c$ & $815490 \pm 50100 b$ & $755750 \pm 27979 b$ & 1 \\
\hline $\begin{array}{l}\text { Kaempferol-3-0-(6"- } \\
\text { acetyl)-glucoside }\end{array}$ & $255677 \pm 29171 a$ & $48383 \pm 6765 b$ & $26931 \pm 2738 b c$ & $16761 \pm 1068 \mathrm{~cd}$ & $4683 \pm 8111 \mathrm{~cd}$ & 0 \\
\hline $\begin{array}{l}\text { Quercetin-0- } \\
\text { acetylhexoside }\end{array}$ & $\mathrm{Ob}$ & $50017 \pm 86631 b$ & $94460 \pm 84150 \mathrm{~b}$ & $324543 \pm 49272 a$ & $293150 \pm 12284 a$ & 2 \\
\hline $\begin{array}{l}\text { Isorhamnetin-acetyl } \\
\text { hexoside }\end{array}$ & $937593 \pm 77367 a$ & $264943 \pm 11698 b$ & $160227 \pm 9203 c$ & $57247 \pm 9497 d$ & $16441 \pm 15156 d$ & 0 \\
\hline $\begin{array}{l}\text { Hesperetin C- } \\
\text { malonylhexoside }\end{array}$ & $37950667 \pm 2265878 a$ & $9506000 \pm 120643 b$ & $8876433 \pm 770626 b$ & $2485000 \pm 253531 \mathrm{c}$ & $1109267 \pm 51664 \mathrm{c}$ & 6 \\
\hline $\begin{array}{l}\text { Tricin 7-O-(6"-O- } \\
\text { Malonyl) -Beta-D- } \\
\text { Glucoside }\end{array}$ & $24980667 \pm 204510 a$ & $8171500 \pm 260968 b$ & $6992133 \pm 575853 b$ & $2804133 \pm 51771 \mathrm{c}$ & $1316667 \pm 96968 c d$ & 5 \\
\hline $\begin{array}{l}\text { Apigenin-7-O-[ }[\beta-D- \\
\text { glucuronosyl }(1 \rightarrow 2)-0 \text { - } \\
\beta \text {-D-glucuronoside) }\end{array}$ & $952890 \pm 39778 a$ & $\mathrm{Ob}$ & $\mathrm{Ob}$ & $\mathrm{Ob}$ & $\mathrm{Ob}$ & 0 \\
\hline $\begin{array}{l}\text { Apigenin-O-rutinoside- } \\
\text { O-Hexoside }\end{array}$ & $1155600 \pm 67073 a$ & $218540 \pm 20069 \mathrm{bc}$ & $336853 \pm 35740 b$ & $151580 \pm 133363 \mathrm{~cd}$ & $37273 \pm 64559 \mathrm{de}$ & 0 \\
\hline
\end{tabular}

In general, in 15 polymethoxyflavones, the content of most of them in PCRC did not have a certain simple trend. However, the contents of polymethoxyflavones in groups with shorter aging periods were lower than those in groups with longer aging periods.

\subsection{Evaluation of total antioxidant activity}

To investigate the impact of the change in flavonoid content in PCRC during the aging process on the bioactivity, FRAP, ABTS, and DPPH methods were employed to determine the total antioxidant activity of the extract solutions of PCRC in six groups with different aging periods (Table 3). The antioxidant potency composite index (APC) was used to evaluate the total antioxidant activity. The results were C3 $>$ C4 $>$ C2 $>$ C29 $>$ C0 $>$ C1, and the APCs of group C3 and group C4 were significantly higher than those of the other four groups, indicating that they had better antioxidant potency. Wang et al.[19] reported that the total phenols of freshly harvested PCRC had the best antioxidant potency, as tested by the peroxyl radical scavenging capacity (PSC) test. PCRC aged for 1 
year and 13 years had the best oxygen radical absorbance capacity (ORAC) test, and PCRC aged for 6 years had the best cellular antioxidant activity (CAA) test.

Table 3

Evaluation of total antioxidant activity of PCRC with different aging periods

\begin{tabular}{|lllll|}
\hline No. & \multicolumn{2}{l|}{ Total antioxidant activity } & IC $_{50}$ & APC \\
\cline { 2 - 5 } & FRAP(mM) & ABTS(mM) & DPPH( $(\mu \mathrm{g} / \mathrm{mL})$ & \\
\hline C0 & $1.66 \pm 0.04 \mathrm{~b}$ & $0.45 \pm 0.01 \mathrm{c}$ & $2.43 \pm 0.43 \mathrm{ab}$ & 38.45 \\
\hline C1 & $1.39 \pm 0.02 \mathrm{~d}$ & $0.55 \pm 0.02 \mathrm{~b}$ & $2.58 \pm 0.10 \mathrm{a}$ & 32.98 \\
\hline C2 & $1.65 \pm 0.08 \mathrm{bc}$ & $0.51 \pm 0.07 \mathrm{bc}$ & $2.45 \pm 0.10 \mathrm{ab}$ & 44.97 \\
\hline C3 & $2.09 \pm 0.15 \mathrm{a}$ & $0.77 \pm 0.01 \mathrm{a}$ & $1.23 \pm 0.08 \mathrm{c}$ & 140.75 \\
\hline C4 & $1.49 \pm 0.05 \mathrm{c}$ & $0.79 \pm 0.03 \mathrm{a}$ & $2.07 \pm 0.41 \mathrm{~b}$ & 86.35 \\
\hline C29 & $1.78 \pm 0.08 \mathrm{~b}$ & $0.44 \pm 0.02 \mathrm{c}$ & $2.48 \pm 0.12 \mathrm{ab}$ & 41.69
\end{tabular}

Note: FRAP/ABTS method use equivalent weight of FeSO $4 \cdot 7 \mathrm{H} 2 \mathrm{O} /$ Trolox, in unit of $\mathrm{mM}$, the larger the stronger potency. DPPH method use $50 \%$ inhibiting concentration $\left(\mathrm{IC}_{50}\right)$, in unit of $\mathrm{mg} / \mathrm{mL}$, the smaller the stronger potency.

\subsection{Molecular docking}

To recognize the effect of PCRC in preventing and treating COVID-19, molecular docking was used to evaluate the binding energy of flavonoids and positive control drugs separately at the protein structures of Spike, 3CLpro, PLpro, and RdRp. A lower binding energy indicates stronger affinity and better potential efficacy. The results of the positive control drugs are shown in Table 4-1. Lopinavir had the lowest binding energy at 3CLpro, RdRp, and spike proteins, with values of $-6.20 \mathrm{kcal} / \mathrm{mol},-10.10 \mathrm{kcal} / \mathrm{mol}$, and $-11.60 \mathrm{kcal} / \mathrm{mol}$, respectively. Ribavirin had the lowest binding energy at the PLpro protein, with a value of -7.30 $\mathrm{kcal} / \mathrm{mol}$.

Table 4 - 2 lists the 32 components from PCRC that had lower binding energy at 3CLpro than lopinavir. Isoschaftoside had the lowest value of $-9.40 \mathrm{kcal} / \mathrm{mol}$. In addition, some flavonoids abundant in PCRC had lower binding energies than lopinavir, e.g., hesperidin - $6.47 \mathrm{kcal} / \mathrm{mol}$, naringin - $7.80 \mathrm{kcal} / \mathrm{mol}$, narirutin $8.87 \mathrm{kcal} / \mathrm{mol}$, neohesperidin $-8.17 \mathrm{kcal} / \mathrm{mol}$, nobiletin $-6.80 \mathrm{kcal} / \mathrm{mol}$, and tangeretin $-6.80 \mathrm{kcal} / \mathrm{mol}$.

Table 4 - 3 lists the 13 components from PCRC that had lower binding energy at RdRp than lopinavir. Linarin had the lowest value of $-11.93 \mathrm{kcal} / \mathrm{mol}$. In addition, some flavonoids abundant in PCRC had lower binding energies than lopinavir, e.g., naringin $-10.77 \mathrm{kcal} / \mathrm{mol}$, narirutin - $11.77 \mathrm{kcal} / \mathrm{mol}$, and neohesperidin $-10.20 \mathrm{kcal} / \mathrm{mol}$.

Table $<$ link rid="tb7">4</link $>-4$ lists the 12 components from PCRC that had lower binding energy at PLpro than ribavirin. Neohesperidin had the lowest value of $-7.83 \mathrm{kcal} / \mathrm{mol}$. In addition, naringin and narirutin had values of $-7.80 \mathrm{kcal} / \mathrm{mol}$ and $7.50 \mathrm{kcal} / \mathrm{mol}$, respectively.

Table 4-5 lists the five components from PCRC that had lower binding energy at spike than lopinavir. Isoxaphoroside had the lowest value of $-13.27 \mathrm{kcal} / \mathrm{mol}$. Naringin and hesperidin had values of $-11.80 \mathrm{kcal} / \mathrm{mol}$ and $11.60 \mathrm{kcal} / \mathrm{mol}$, respectively.

To compare the potential antiviral activities of PCRC with different aging periods against SARS-CoV-2, the total content of flavonoids with lower binding energy than the positive control drug was added up for separate targeting proteins and separate aging periods. Figure 9 a shows that the total content of 32 flavonoids with lower binding energy than the positive control drug at $3 \mathrm{CLpro}$ varied with the aging period. It was found that the content of group CO was highest. Of the rest of the groups, the content of group C3 was the highest. Figure 9b, 9c, and 9d show the results for the binding target proteins RdRp, PLpro, and spike, respectively. Generally, the total content of flavonoids with lower binding energy than the positive control drug was highest in group C0, decreased in the one-year aging process, increased to the second-highest level, and decreased as the aging period extended.

Table 4

-1 Molecular docking result of positive control drugs

\begin{tabular}{|lllll|}
\hline positive control drug & \multicolumn{4}{c|}{ Binding energy(kcal/mol) } \\
\cline { 2 - 5 } & 3CLpro & RdRp & PLpro & Spike protein \\
\hline Lopinavir & -6.20 & -10.10 & -6.23 & -11.60 \\
\hline Ritonavir & -6.13 & -8.67 & -6.20 & -9.37 \\
\hline Ribavirin & -5.90 & -7.70 & -7.30 & -7.50 \\
\hline Chloroquine & -6.10 & -6.97 & -5.07 & -7.13 \\
\hline Arbidol & -6.03 & -8.03 & -5.00 & -7.00 \\
\hline Favipiravir & -4.73 & -6.20 & -6.43 & -6.07 \\
\hline
\end{tabular}


Table 4

-2 Molecular docking results of flavonoids at 3CLpro

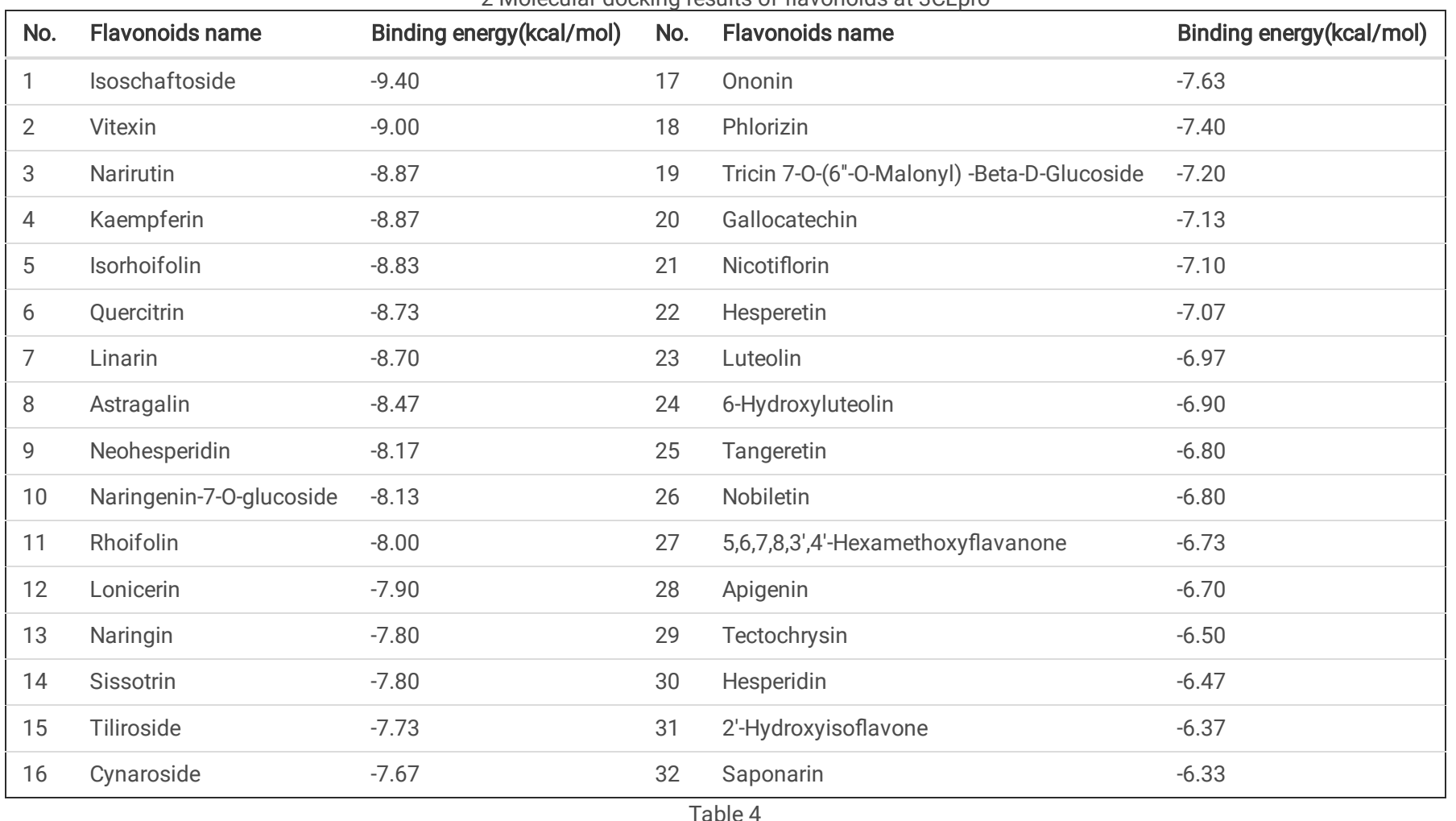

-3 Molecular docking results of flavonoids at RdRp

\begin{tabular}{|llllll|}
\hline No. & Flavonoids name & Binding energy(kcal/mol) & No. & Flavonoids name & Binding energy(kcal/mol) \\
\hline 1 & Linarin & -11.93 & 8 & Rhoifolin & -10.73 \\
\hline 2 & Isorhoifolin & -11.80 & 9 & Saponarin & -10.70 \\
\hline 3 & Narirutin & -11.77 & 10 & Hesperetin & -10.40 \\
\hline 4 & Lonicerin & -11.13 & 11 & Neohesperidin & -10.20 \\
\hline 5 & Isoschaftoside & -11.10 & 12 & Gallocatechin & -10.07 \\
\hline 6 & Nicotiflorin & -11.00 & 13 & Cynaroside & -10.07 \\
\hline 7 & Naringin & -10.77 & & & \\
\hline
\end{tabular}

Table 4

4 Molecular docking results of flavonoids at PLpro

\begin{tabular}{|llllll|}
\hline No. & Flavonoids name & Binding energy(kcal/mol) & No. & Flavonoids name & Binding energy(kcal/mol) \\
\hline 1 & Neohesperidin & -7.83 & 7 & Astragalin & -7.57 \\
\hline 2 & Naringin & -7.80 & 8 & Kaempferin & -7.53 \\
\hline 3 & Quercitrin & -7.80 & 9 & Narirutin & -7.50 \\
\hline 4 & Isorhoifolin & -7.67 & 10 & Cynaroside & -7.33 \\
\hline 5 & Linarin & -7.63 & 11 & Nicotiflorin & -7.33 \\
\hline 6 & Lonicerin & -7.57 & 12 & Gallocatechin & -7.33 \\
\hline
\end{tabular}

Table 4

5 Molecular docking results of flavonoids at Spike

\begin{tabular}{|llllll|}
\hline No. & Flavonoids name & Binding energy(kcal/mol) & No. & Flavonoids name & Binding energy(kcal/mol) \\
\hline 1 & Isoschaftoside & -13.27 & 4 & Rhoifolin & -11.80 \\
\hline 2 & Lonicerin & -11.83 & 5 & Isorhoifolin & -11.67 \\
\hline 3 & Naringin & -11.80 & 6 & Hesperidin & -11.60 \\
\hline
\end{tabular}




\section{Discussion}

In the latest report, 92 flavonoids were detected from the methanol extract solution of PCRC [23], and up to 56 polymethoxyflavones were identified from PCRC [28]. The marker components of PCRC recorded in the Chinese Pharmacopeia (2020 version, One Sections) were hesperidin, nobiletin, and tangeretin [1]. Studying the main components, hesperidin, nobiletin, and tangeretin, in PCRC were beneficial to the quality evaluation of PCRC [19-23]. However, in the aging process, the contents of polyphenols, flavonoids, and particularly polymethoxyflavones undergo a series of changes [19, 23]. Therefore, investigating the overall change in flavonoids in the aging process of PCRC is a prerequisite and guarantees reasonable quality control and standard establishment for PCRC. In this study, PCRC samples from the same tree (excluding the group of aging period of 29 years), same farm, and stored under the same conditions were used as objects. The total flavonoid contents of PCRC from six groups with different aging periods were determined by spectrophotometry. The results showed that with increasing aging time, the total flavonoid contents of PCRC decreased at first, then increased, and then decreased again. Furthermore, UPLC-MS/MS and widely targeted metabolomics analysis were employed to intensively investigate the composition of the flavonoids in PCRC with different aging periods. Up to 219 metabolites of flavonoids were detected in all samples, including 82 flavones, 56 flavonols, 26 flavonoid C-glycosides, 19 dihydroflavones, 7 isoflavones, 7 tannins, 5 flavanols, 5 dihydroflavonols, 4 anthocyanins, 3 chalcones, 2 other flavonoids, 2 proanthocyanidins, and 1 dihydroisoflavone. Among them, two flaonoids, 5,7,3',4',5'-pentamethoxy- dihydroflavones and 2'-hydroxy-3,4,5,3',4',6'-hexamethoxychalcone, were detected in PCRC for the first time. By clustering analysis, it was found that samples of freshly harvested PCRC and PCRC with aging periods of 1 year and 2 years clustered into one class. Samples of PCRC with an aging period of 3 years and over 3 years clustered into the other class. This result indicated that qualitative and quantitative changes occurred in the component composition of PCRC with an aging period of over 3 years. Further analysis reviewed that polymethoxyflavones in groups with longer aging periods $(3,4,29$ years) were more abundant than those in groups with shorter aging periods $(0,1,2$ years). Polymethoxyflavones are one kind of important component in PCRC. Because their molecular structures are more lipophilic, they penetrate biofilms more easily and enhance bioavailabilities. Therefore, the bioactivity of polymethoxyflavones was better than that of the relevant structure without methoxylation [29, 30]. Polymethoxyflavones not only have good antioxidative activity but also have an effect on relieving metabolic syndromes such as hypertriglyceridemia, fatty liver, and insulin resistance. by regulating enteric microorganisms and amino acid metabolism [22,31,32]. They have antitumor effects by inhibiting the growth of tumor cells and inducing the apoptosis of tumor cells $[29,30,33]$. The results reviewed the aging process to enhance the compound number and total content of the polymethoxyflavones in PCRC and further indicated the scientific meaning of "the longer the aging period is, the better" for PCRC.

However, regarding the antioxidative effect, the potency of PCRC in the groups aged 3 years was better than that in the other 5 groups. The group with the longest aging period of 29 years did not present a significant advantage. Its potency was only equal to that of the group with an aging period of 2 years. The results explained the record of an aging period of $2 \sim 3$ years in a medical book written by Yuhe Xu in the Qing Dynasty [34].

Of all the differential flavonoid metabolites in the five pairs of PCRC groups, C0 vs C1, C0 vs C2, C0 vs C3, C0 vs C4, and C0 vs C29, 21 were the same. Seven flavonoids (tectochrysin, apigenin, 2'-hydroxyisoflavone, luteolin, 6-hydroxyluteolin, gallocatechin, quercetin-0-acetylhexoside) were detected in five groups of aging PCRC samples and were not detected in newly harvested PCRC samples. Apigenin-7-O-[ $\beta$-D-glucuronide $(1 \rightarrow 2)-0-\beta-D-$ glucuronide] was only detected in freshly harvested PCRC samples. This result indicated that the aging process might prompt the synthesis or decomposition of some flavonoids by initiating special biochemical metabolic processes. It was reported that some key microbes associated with $C$. reticulata 'Chachi' could be involved in the improvement of its health properties [34]. In addition, 5,7,3',4',5'- pentamethoxy dihydroflavone and 2'- hydroxy-3,4,5,3'4',6'- hexamethoxychalcone were found from PCRC for the first time. Their pharmacological activity and possible application deserve further study. Seven flavonoids appeared, and flavonoids disappeared in the aging process, which might be the main object components for later studies relating to flavonoid metabolism regulated by microbes in the aging process.

Currently, COVID-19 is still prevalent around the world. Although vaccines were invented, there is still much uncertainty. New mutated viruses emerged and led to an enhanced ability to spread and continue to deteriorate the epidemic situation in some local areas. Finding active compounds to prevent and/or treat COVID-19 is important and urgent. Some flavonoids were reported to interact with some targets of SARS-CoV-2[27]. Interfering with the spike protein of SARSCoV-2 and the ACE2 receptor of the host or inhibiting RNA polymerase and important proteases (3CLpro and PLpro) inhibit virus reproduction and might be a potential treatment for COVID-19[27]. Molecular docking was performed using flavonoids from PCRC targeting the spike protein, 3CLpro, PLpro, and RdRp of SARS-CoV-2. It was found that many flavonoids from PCRC had better affinity for the target than positive control drugs. The total content of flavonoids with lower binding energy than the positive control drug was highest in newly harvested PCRC. This result indicated that newly harvested PCRC would be more appropriate for the preparation to prevent or treat COVID-19. The results are only based on molecular docking. In vivo and in vitro experiments should be further carried out to evaluate the antiviral activity of PCRC flavonoids against SARS-CoV-2.

\section{Conclusions}

UPLC-MS/MS and metabolomics analysis were employed to analyze the flavonoids of PCRC with different aging periods for the first time. The results showed that the aging process prompted qualitative and quantitative changes in flavonoids in PCRC, and polymethoxyflavone significantly increased in PCRC aged for 3,4 , and 29 years. However, the potential antiviral components against SARS-CoV-2 decreased as the aging period extended. The results of APC experiments showed that PCRC with aging periods of 3 and 4 years presented significantly higher antioxidative potency than the other groups, and PCRC with an aging period of 29 years did not present an antioxidative advantage. Therefore, it was recommended to use PCRC with an aging period over 3 years according to the content of bioactive polyemethoxyflavones, and for antioxidative use, PCRC with an aging period of $3 \sim 4$ years would be better. For use in preparation preventing or treating COVID-19, newly harvested PCRC would be more appropriate. In addition, 5,7,3',4',5'-pentamethoxy dihydroflavone and 2'-hydroxy$3,4,5,3^{\prime} 4^{\prime}, 6^{\prime}$ - hexamethoxychalcone were found from PCRC for the first time. At the same time, seven flavonoids (tectochrysin, apigenin, 2'-hydroxyisoflavone, luteolin, 6-hydroxyluteolin, gallocatechin, quercetin-0-acetylhexoside) were detected in aging PCRC samples, and apigenin-7-0-[ $\beta$-D-glucuronide (1 $\rightarrow 2$ )-0- $\beta$-Dglucuronide] disappeared after aging. The results in this study supplied scientific guidance information for fully evaluating component changes in PCRC with different aging periods and rational utilization in medicine and supplied important clues for intensively investigating the synthesis of related compounds and the regulatory mechanism of metabolism in the aging process. 


\section{Abbreviations}

PCRC

Pericarp of Citri Reticulatae 'Chachi'; UPLC-MS/MS:Ultra-performance liquid chromatography coupled with triple quadrupole mass spectrometry; APC:Antioxidant potency composite index; WHO:World Health Organization; QC:Quality control; FRAP:Ferric reducing ability of plasma; ABTS:2,2'-Azinobis-(3ethylbenzthiazoline-6-sulphonate); DPPH:1,1-Diphenyl-2-picrylhydrazyl; LC-ESI-MS/MS:High performance liquid chromatography-electrospray tandem mass spectrometry; QQQ:Triple quadrupole; Q TRAP:Triple quadrupole-linear ion trap; LC/MS/MS:Liquid chromatography-tandem mass spectrometry; IS:Ion spray voltage; GSI:Ion source gas I; GSII:Gas II; CUR:Curtain gas; CAD:Collision gas; MRM:Multiple reaction monitoring; COVID-19:Coronavirus Disease 2019; PCA:Principal component analysis; HCA:Hierarchical cluster analysis; PCC:Pearson correlation coefficients; OPLS-DA:Orthogonal Partial Least Squares Discrimination Analysis; KEGG:Kyoto Encyclopedia of Genes and Genomes; MSEA:Metabolite set enrichment analysis; TIC:Total ion current; VIP:Variance importance P value; PSC:Peroxyl radical scavenging capacity; ORAC:Oxygen radical absorbance capacity; $I_{50}: 50 \%$ inhibiting concentration.

\section{Declarations}

\section{Acknowledgements}

Not applicable.

\section{Authors' contributions}

HW and SJL conceived and designed the project; ZJW,YYL,FLD and TXX conducted the experiemnt and analysed the results; HW, SJL and TXT analysed the data and wrote the paper.

\section{Funding}

This project was financially supported by the Key realm R\&D Program of Guangdong Province区2020B020221001)هThe National Natural Science Foundation

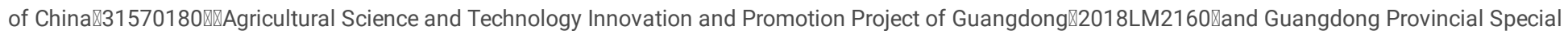
Fund for Modern Agriculture Industry Technology Innovation Teams(2019KJ125)凶Fund of National Modern Agricultural Industrial Park of Xinhui Tangerine Peel.

\section{Availability of data and materials}

The original data generated from this study are accompanied with the article as additional files.

\section{Declarations}

\section{Ethics approval and consent to participate}

Not applicable.

\section{Consent for publication}

Not applicable.

\section{Competing interests}

The authors declare that they have no financial and personal relationships with other people or organizations that can inappropriately influence their work, and that there is no professional or other personal interest of any nature or kind in any product, service and/or company that could be construed as influencing the position presented in, or the review of, this paper.

\section{Author details}

${ }^{1}$ State Key Laboratory for Conservation and Utilization of Subtropical Agro-bioresources, South China Agricultural University, Guangzhou 510642,China. ${ }^{2}$ College of Life Science, South China Agricultural University, Guangzhou 510642,China.

${ }^{3}$ Zhaoqing Medical College, Zhaoqing 526060, China. ${ }^{4}$ Jiaying University, Meizhou 514015, China.

\section{References}

1. National Pharmacopoeia Commission. Pharmacopoeia of the People's Republic of China, One Sections. Beijing: China Medical Science and Technology Press; 2020. pp. 199-200.

2. Luo Y, Zeng W, Huang KE, Li DX, Chen W, Yu XQ, Ke XH. Discrimination of Citrus reticulata Blanco and Citrus reticulata'Chachi'as well as the Citrus reticulata'Chachi'within different storage years using ultra high performance liquid chromatography quadrupole/time-of-flight mass spectrometry based metabolomics approach. J Pharm Biomed Anal. 2019;171:218-31. https://doi.org/10.1016/j.jpba.2019.03.056.

3. Fu MQ, An KJ, Xu YJ, Chen YL, Wu Jj, Yu YS, Zou B, Xiao GS, Ti HH. Effects of different temperature and humidity on bioactive flavonoids and antioxidant activity in pericarpium Citri reticulata (Citrus reticulata 'chachi'). LWT-Food Science Technology. 2018;93:167-73. 
https://doi.org/10.1016/j.Iwt.2018.03.036.

4. Liu EH, Zhao P, Duan L, Zheng GD, Guo L, Yang H, Li P. Simultaneous determination of six bioactive flavonoids in Citri reticulatae pericarpium by rapid resolution liquid chromatography coupled with triple quadrupole electrospray tandem mass spectrometry. Food Chem. 2013;141(4):3977-83. https://doi.org/10.1016/j.foodchem.2013.06.077.

5. Xu JJ, Wu X, Li MM, Li GQ, Yang YT, Luo HJ, Huang WH, Chung HY, Ye WC, Wang GC, Li YL. Antiviral activity of polymethoxylated flavones from "Guangchenpi", the edible and medicinal pericarps of citrus reticulata'Chachi'. J Agric Food Chem. 2014;62(10):2182-9. https://doi.org/10.1021/jf404310y.

6. Gao B, Chen YL, Zhang MW, Xu YJ, Pan SY. Chemical Composition, Antioxidant and Antimicrobial Activity of Pericarpium Citri Reticulatae Essential Oil. Molecules.2011;16(5):4082-4096. https://doi.org/10.3390/molecules16054082.

7. Chen XM, Tait AR, Kitts DD. Flavonoid composition of orange peel and its association with antioxidant and anti-inflammatory activities. Food Chem. 2017;218:15-21. https://doi.org/10.1016/j.foodchem.2016.09.016.

8. Yu JJ, Su J, Yan MQ, Lou ZH, Lyu GY. Correlation between lipid-lowering efficacy and components of pericarpium Citri reticulatae. China J Chin Materia Med. 2019;44(15):3335-42. https://doi.org/10.19540/j.cnki.cjcmm.20190523.304.

9. Zeng W, Luo Y, Huang KE, Chen W, Yu XQ, Li DX, Ke XH. Serum Metabolomics of Hyperlipidemia Intervened by Citri Reticulatae Chachiensis Pericarpium. Chinese medicine new drugs and clinical pharmacology. 2020;31(01):72-79. https://doi.org/10.19378/j.issn.1003-9783.2020.01.011.

10. Gu GN. Shennong's Herbal Classic. Harbin: Harbin Press; 2007. pp. 71-2.

11. Wang J, Huang QW, Zhang YL, Su J, Liu J. Current Situation of the Long-stored TCM Research and Exploration of the Ideas. China Pharmacy. 2012;23(15):1433-5. https://doi.org/10.6039/j.issn.1001-0408.2012.15.36.

12. Sun SM. Qian Jin Shi Zhi. Beijing: China Commercial Press; 1985. p. 24.

13. Su S. Bencao Tujing. Fuzhou: Fujian Science and Technology Press; 1988. p. 482.

14. Wang HG. Tangye Bencao. Beijing: People's Health Publishing House. 1987.p.156.

15. Du WX. YaoJian. Beijing: Chinese Medicine Press.1993.p.46.

16. Ni ZM. Collected Statements on the Herbal Foundation. Beijing: Ancient Books of Traditional Chinese Medicine Press; 2005. p. 570.

17. Wang A. Bencao Beiyao. Beijing: Chinese Medicine Press; 1998. p. 187.

18. Wang ZL, Zhang X, Liu SJ, Chen HP, Liu YP. Historical Evolution and Research Status of Citri Reticulatae Pericarpium. Chinese Archives of Traditional Chinese Medicine. 2017;35(10):2580-4. https://doi.org/10.13193/j.issn.1673-7717.2017.10.031.

19. Wang H, Chen G, Fu X, Liu RH. Effects of aging on the phytochemical profile and antioxidative activity of Pericarpium Citri Reticulatae'Chachiensis'. RSC Advances. 2016;107:105272-81. https://doi.org/10.1039/C6RA22082G.

20. Liu LN, Xu YJ, Xiao GS, Wu JJ, Yu YS, Fu MQ. Flavonoids and antioxidant activity of Pericarpium Citri Reticulatae in different storage years. Journal of Southern Agriculture. 2020;51(03):623-9. https://doi.org/10.3969/j.issn.2095-1191.2020.03.019.

21. Zheng GD, Jiang L, Yang X, Yang DP, Zhou F, Lin LW. Study on the contents of flavonoids in Citrus reticulata'Chachi'storaged in different yearss. Chinese Traditional Patent Medicine. 2010;32:977-80. https://doi.org/10.3969/j.issn.1001-1528.2010.06.026.

22. Fu MQ, Xu YJ, Chen YL, Wu JJ, Yu YS, Zou B, An KJ, Xiao GS. Evaluation of bioactive flavonoids and antioxidant activity in Pericarpium Citri Reticulatae (Citrus reticulata 'Chachi') during storage. Food Chem. 2017;230:649-56. https://doi.org/10.1016/j.foodchem.2017.03.098.

23. Zhang J, Wu XF, Qiu JQ, Zhang L, Zhang YT, Qiu XH, Huang ZH, Xu W. Comprehensive Comparison on the Chemical Profile of Guang Chen Pi at Different Ripeness Stages Using Untargeted and Pseudotargeted Metabolomics. J Agric Food Chem. 2020;68:8483-95. https://doi.org/10.1021/acs.jafc.0c02904.

24. Wu CR, Liu Y, Yang YY, Zhang P, Zhong W, Wang YL, Wang QQ, Xu Y, Li MX, Li XZ, Zheng MZ, Chen LX, Li H. Analysis of therapeutic targets for SARS-CoV-2 and discovery of potential drugs by computational methods. Acta Pharmaceutica Sinica B. 2020;10(5):766-88.

https://doi.org/10.1016/j.apsb.2020.02.008.

25. Meyers KJ, Watkins CB, Pritts MP, Liu RH. Antioxidant and antiproliferative activities of strawberries. J Agric Food Chem. 2003;51:6887-92. https://doi.org/10.1021/jf034506n.

26. Kong DX, Li YQ, Bai M, Deng YL, Liang GX, Wu H. A comparative study of the dynamic accumulation of polyphenol components and the changes in their antioxidant activities in diploid and tetraploid Lonicera japonica. Plant Physiology Biochenmistry. 2017;112:87-96.

https://doi.org/10.1016/j.plaphy.2016.12.027.

27. National Health Commission of the people's Republic of China. Diagnosis and treatment plan for COVID-19(trial version 8). Chinese Journal of Clinical Infectious Diseases. 2020;13(5):321-8. https://doi.org/10.3760/cma.j.issn.1674-2397.2020.05.001.

28. Zeng SL, Duan L, Chen BZ, Li P, Liu EH. Chemicalome and metabolome profiling of polymethoxylated flavonoids in Citri reticulatae pericarpium based on an integrated strategy combining background subtraction and modified mass defect filter in a microsoft excel platform. Journal of Chromatograph A. 2017;1508:106-20. https://doi.org/10.1016/j.chroma.2017.06.015.

29. Hung WL, Chang WS, Lu WC, Wei GJ, Wang Y, Ho CT, Hwang LS. Pharmacokinetics, bioavailability, tissue distribution and excretion of tangeretin in rat. Journal of Food Drug Analysis. 2018;26(2):849-57. https://doi.org/10.1016/j.jfda.2017.08.003.

30. Li RW, Theriault AG, Au K, Douglas TD, Casaschi A, Kurowska EM, Mukherjee R. Citrus polymethoxylated flavones improve lipid and glucose homeostasis and modulate adipocytokines in fructose-induced insulin resistant hamsters. Life Sci. 2006;79(4):365-73.https://doi.org/ 10.1016/j.Ifs.2006.01.023.

31. Ding CG, Sun SQ, Zhou Q, Zhang JL. Chemical fingerprint analysis of tangerine peel reserved for different years by HPLC-DAD and HPLC-HRMS. Chinese Journal of new drugs. 2008;11:927-30. https://doi.org/10.3321/j.issn:1003-3734.2008.11.008. 
32. Zeng SL, Li SZ, Xiao PT, Cai YY, Chu C, Chen BZ, Li P, Li J, Liu EH. Citrus polymethoxyflavones attenuate metabolic syndrome by regulating gut microbiome and amino acid metabolism. Science Advances. 2020;6(1):eaax6208. https://doi.org/10.1126/sciadv.aax6208.

33. Whitman SC, Kurowska EM, Manthey JA, Daugherty A. Nobiletin, a citrus flavonoid isolated from tangerines, selectively inhibits class A scavenger receptor-mediated metabolism of acetylated LDL by mouse macrophages. Atherosclerosis. 2005;178(1):25-32.

https://doi.org/10.1016/j.atherosclerosis.2004.07.034.

34. He J, Chen CC, He QX, Li JY, Ying F, Chen G. The central bacterial community in Pericarpium Citri Reticulatae 'Chachiensis'. Food Res Int. 2019;125:108624. https://doi.org/10.1016/j.foodres.2019.108624.

\section{Figures}

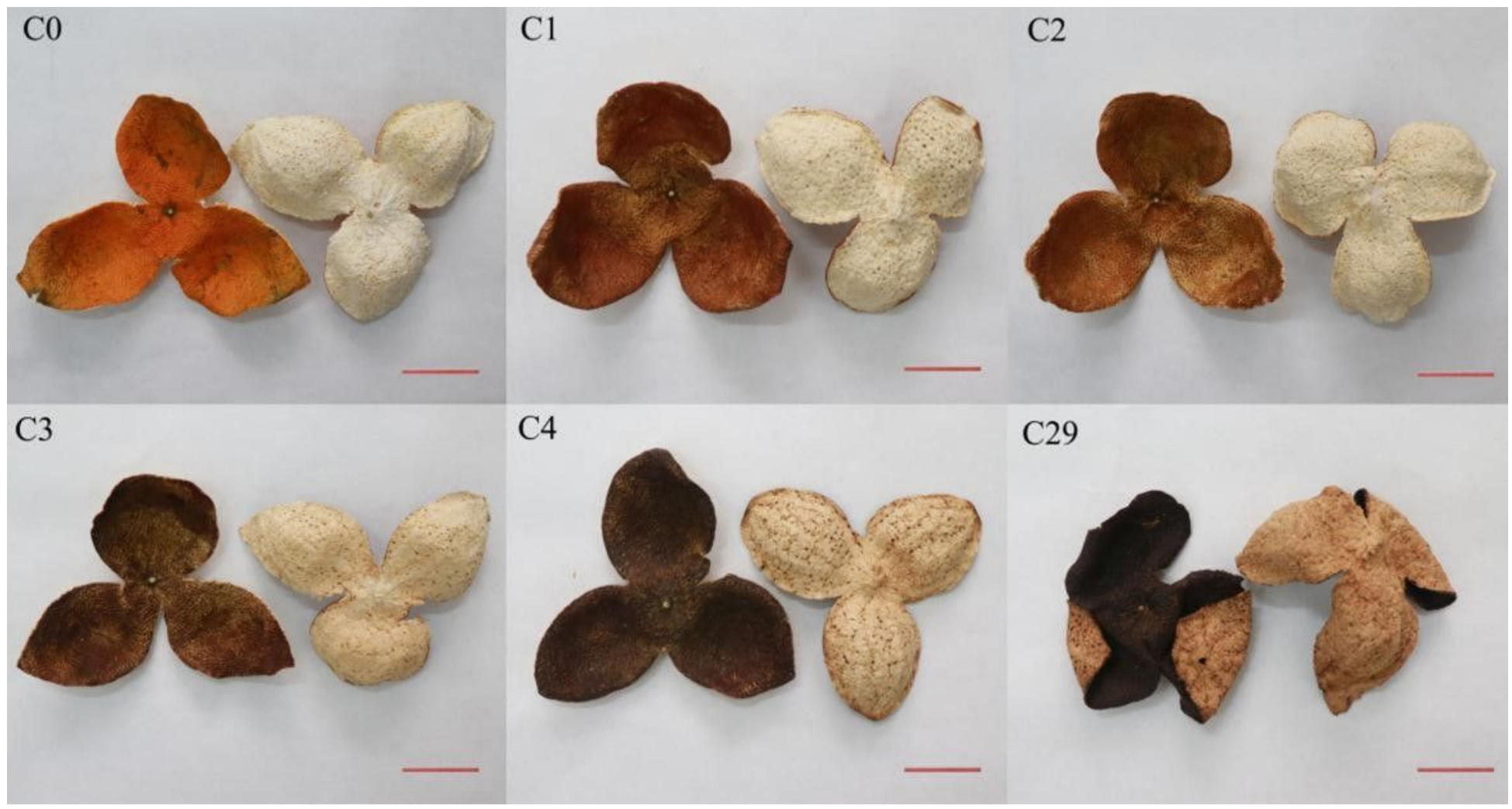

Figure 1

Images of the PCRC samples Bars $=2 \mathrm{~cm}$. 


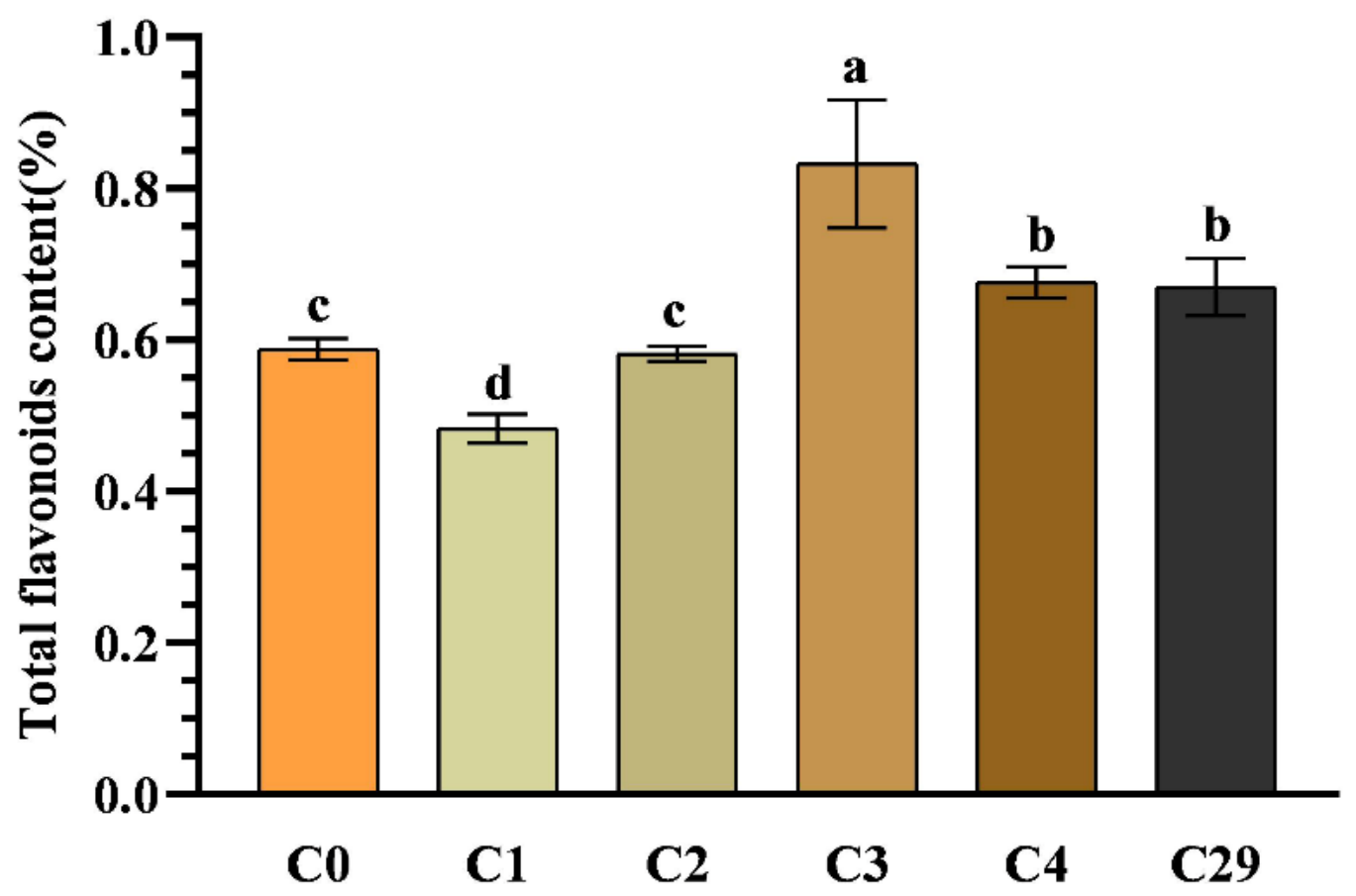

Figure 2

Total flavonoid content of PCRC with different aging peroid Note: Total flavonoid content (with rutin as the standard)= concentration of total flavonoid in extract solution of PCRC*volume of solution* $100 \%$ /weight of PCRC sample

a

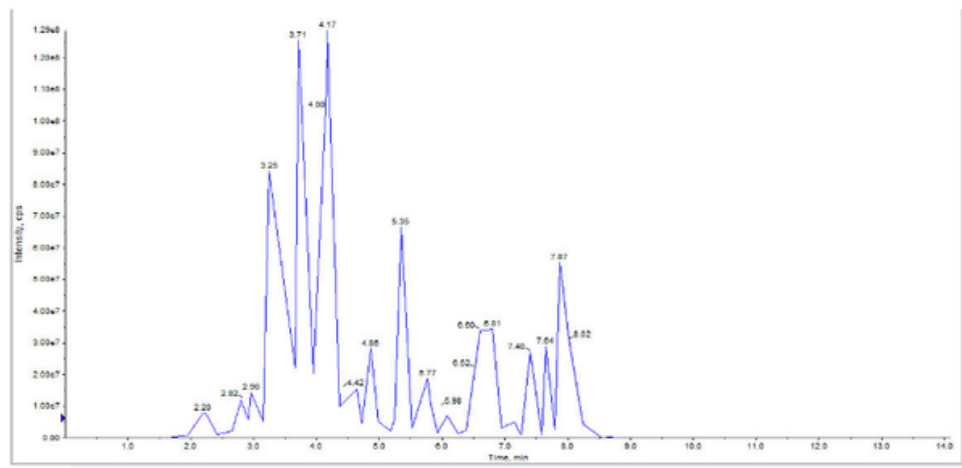

c

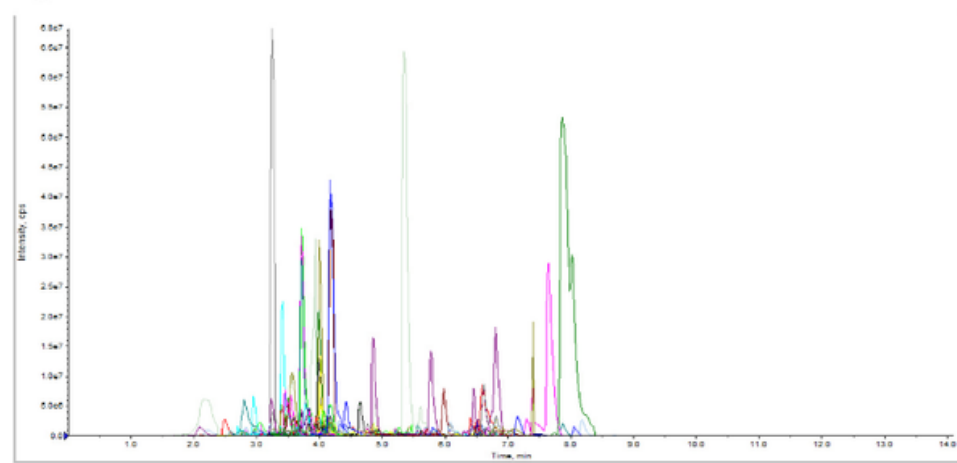

b

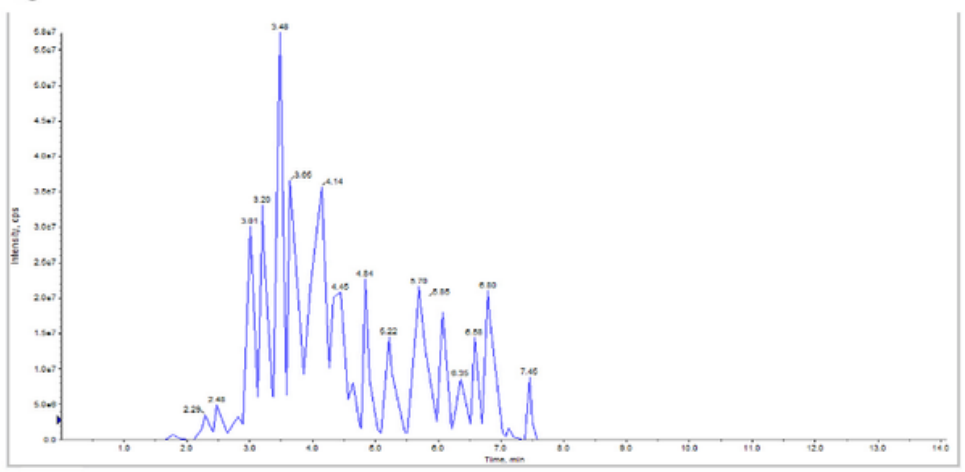

d

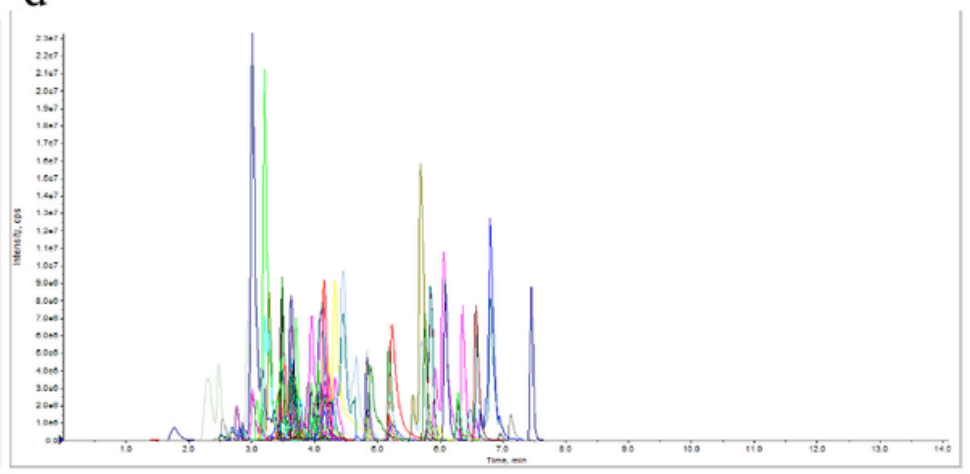

Figure 3

TIC of quality control samples and multi-peaks chromatograms of metabolites using multiple reactions monitoring a, b shows positive TIC chromatoram and negative TIC chromatogram of quality control samples. c, $d$ shows positive and negative ion current multi-peaks chromatograms of meatbolites in quality control samples using MRM. Horizontal ordinate represents retention time of detected metabolites, vertical coordinate represents the ion current strength of detected ion. 


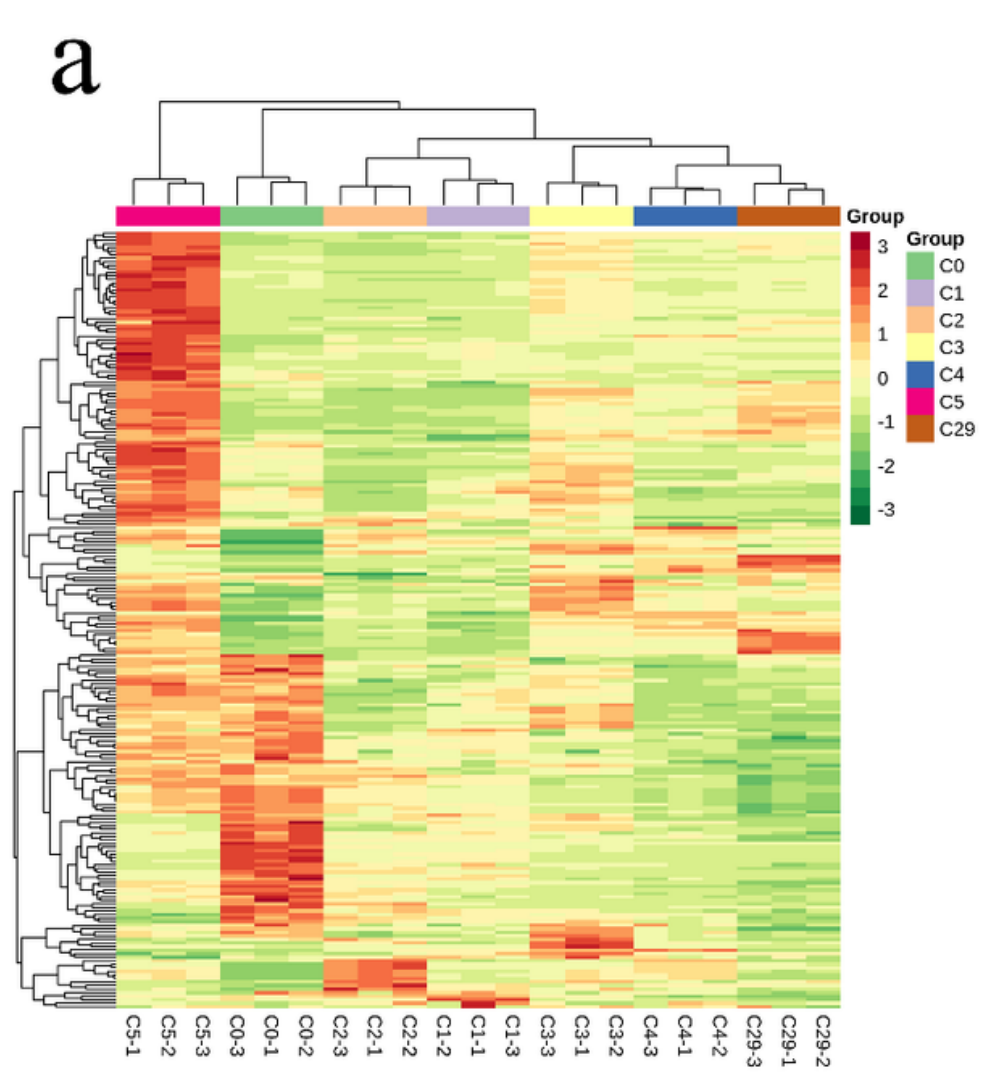

\section{b}

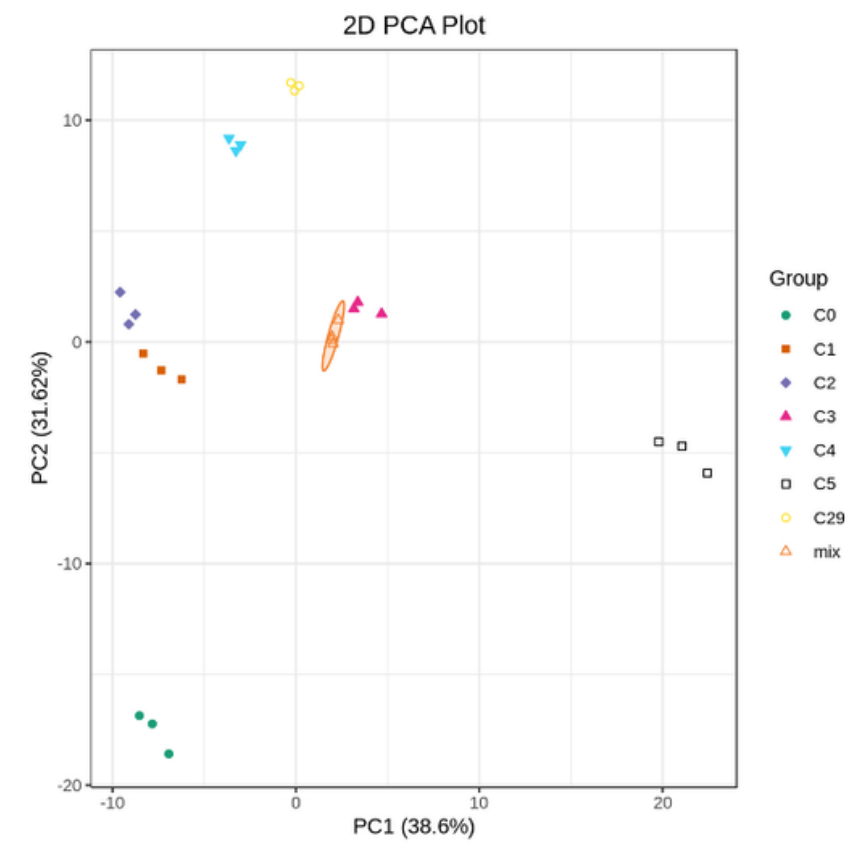

Figure 4

Differece of the flavonoid metabolites in groups of PCRC $₫ a \rrbracket C l$ lustering analysis of flavonoid metabolites in 6 groups of PCRC. The accumulation level of metabolites from high to low was indicated by colors from red to green. खb囚PCA of flavonoid metabolites in 6 groups of PCRC. Horizontal ordinate shows PC1 value, and vertical coordinate shows PC2 value. 


\section{C0 vs C1}

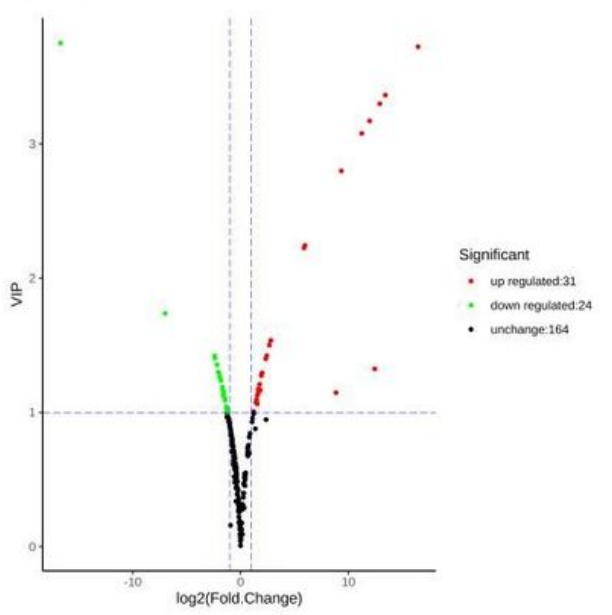

$\mathrm{CO}$ vs C2

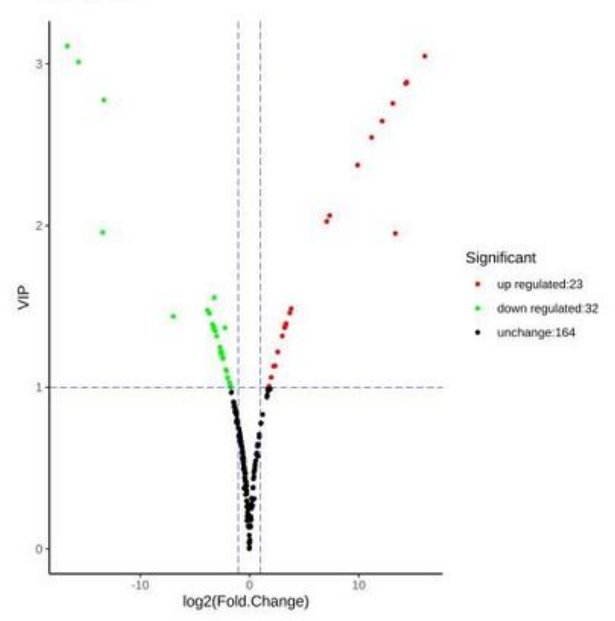

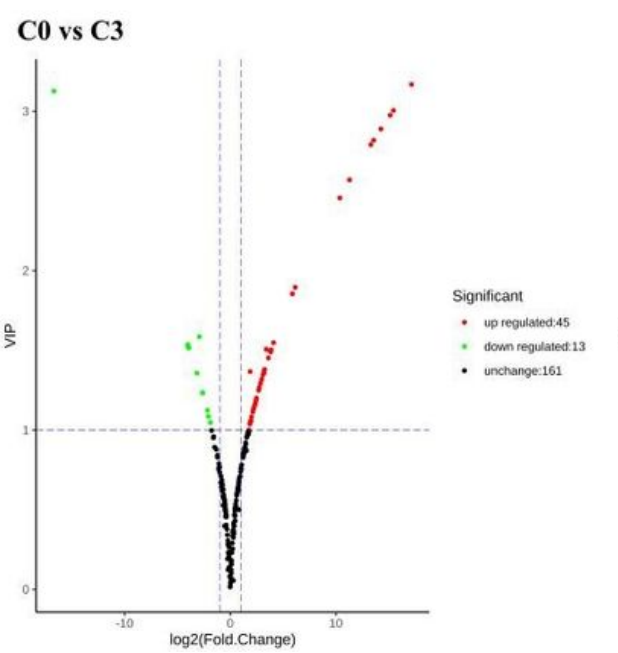
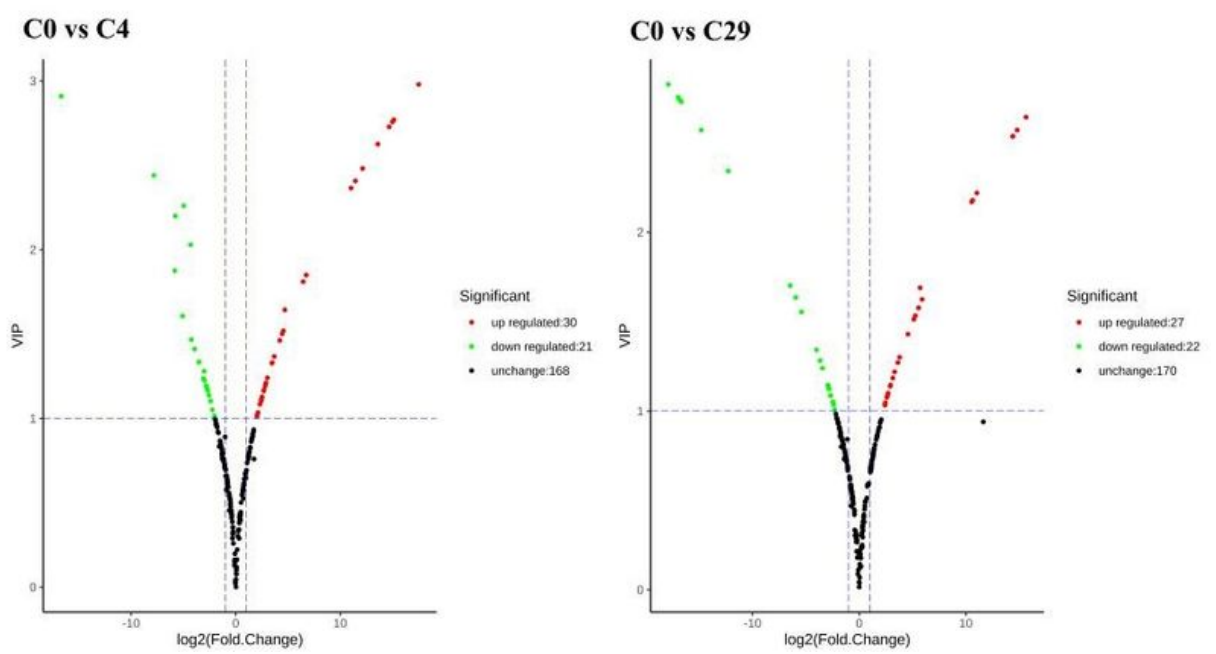

Figure 5

Volcano diagram of differential metabolites Note: Every point in the volcano diagram represents a metabolite. Horizontal ordinate represents the logarithmic value of quantitative fold change of a certain metabolites between two groups of samples. Vertical coordinate represents VIP value. The greater absolute value of horizontal ordinate indicates the bigger fold change of expression amount between two groups of samples. The greater absolute value of vertical coordinate indicates the expression change is more significant and the differential metabolite found by screening is more convincing. The green point indicates the expression of the differential metabolite down-regulated. The red point indicates the expression of the differential metabolite up-regulated. The black point indicates the expression of the differential metabolite is not significant. 


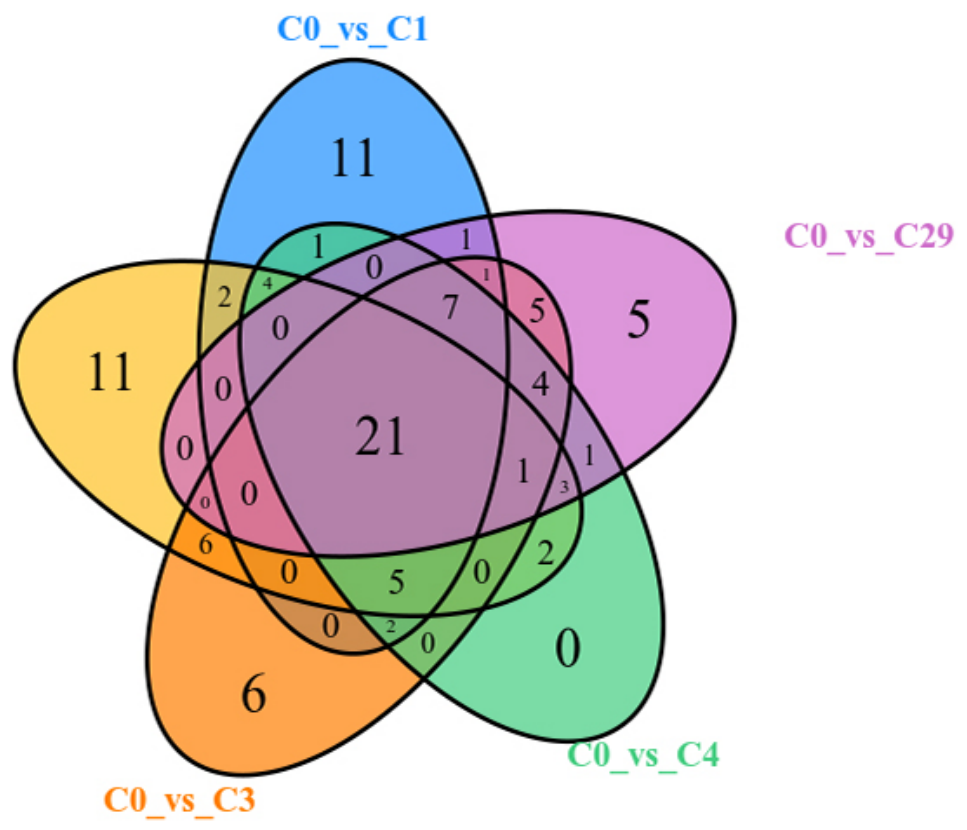

Figure 6

Venn Diagram of differential flavonoid metabolites in five pair groups for compariso

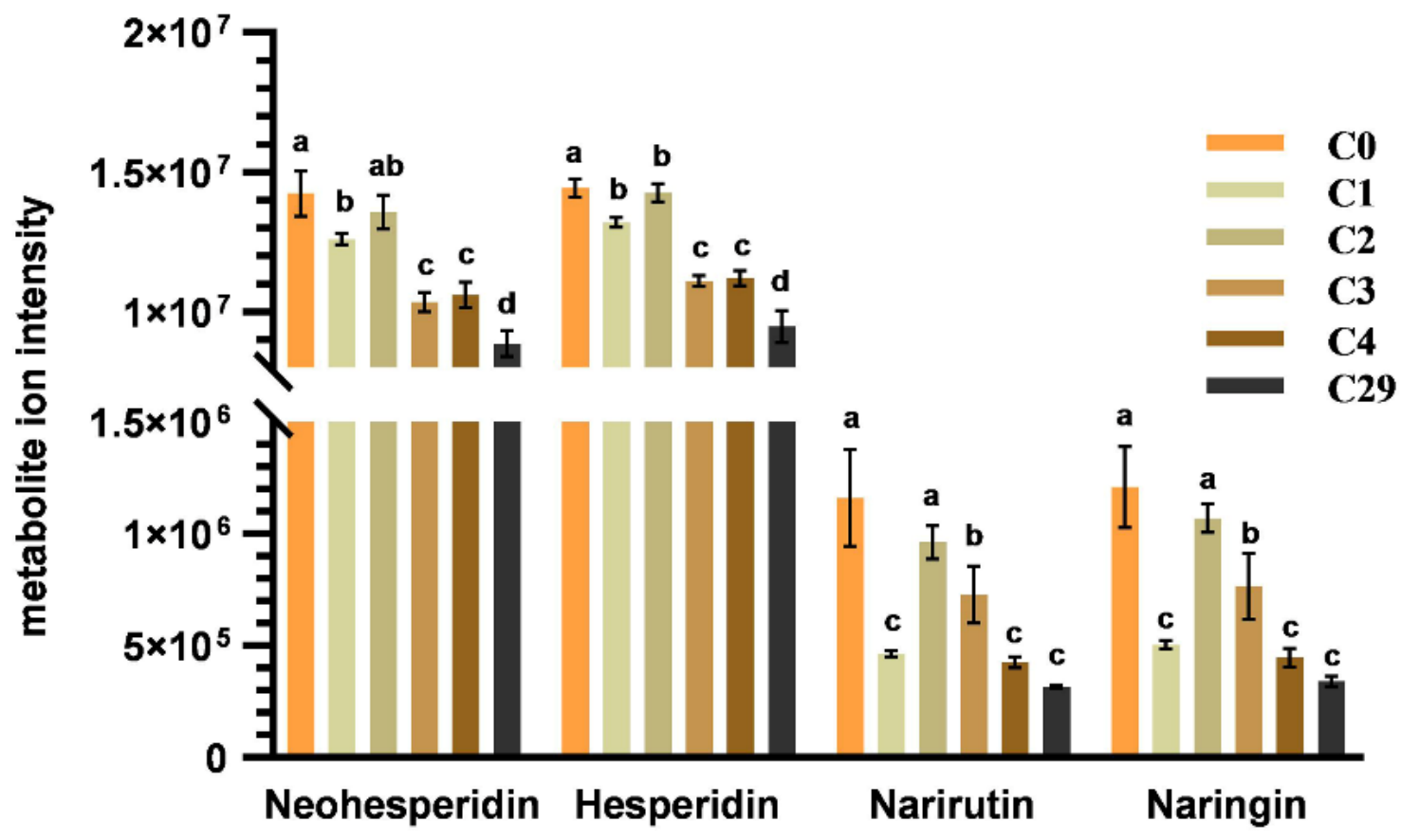

Figure 7

The change of contents of four flavonoid glycosides 


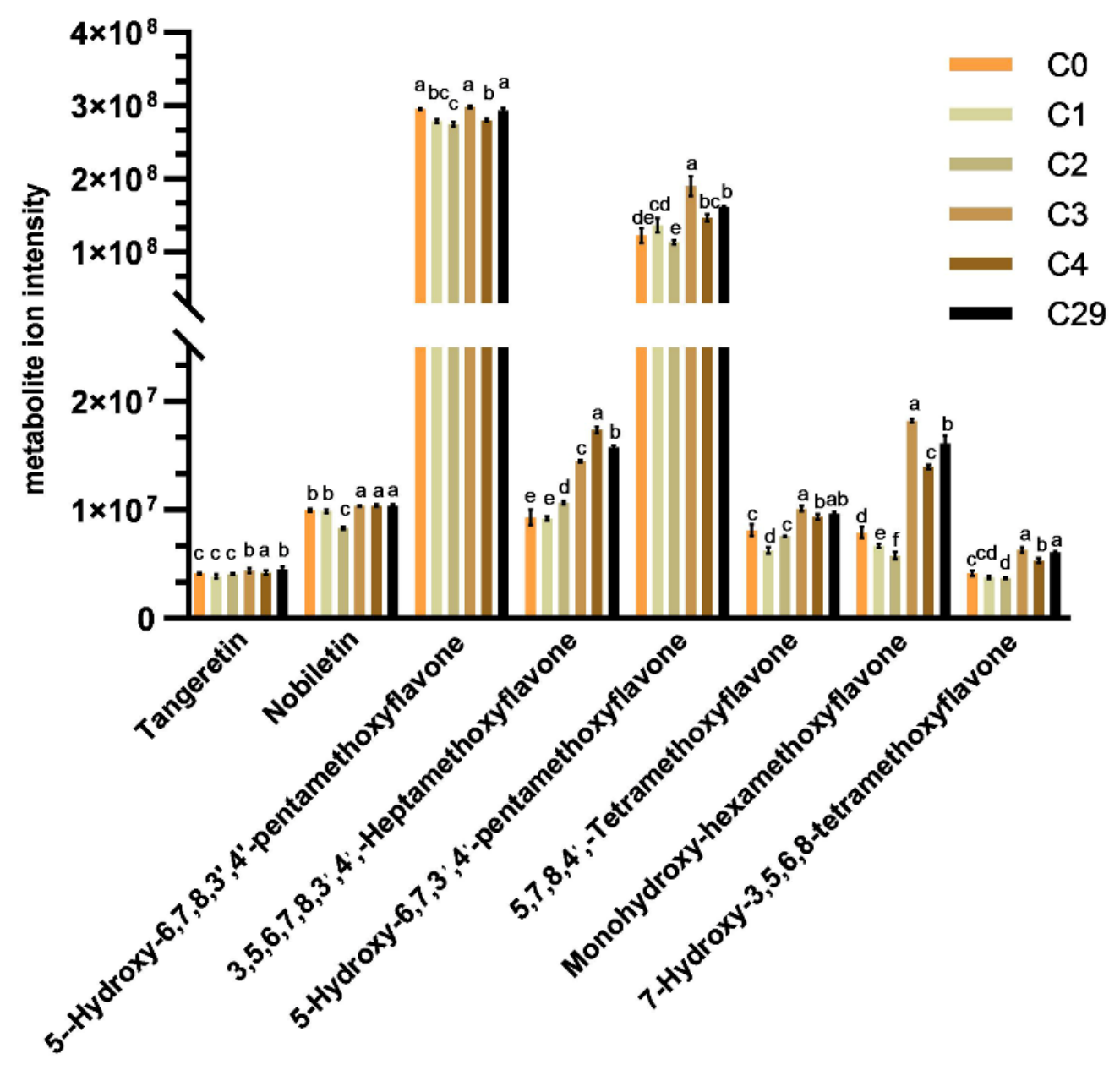

Figure 8

The change of contents of eight polymethoxyflavones 


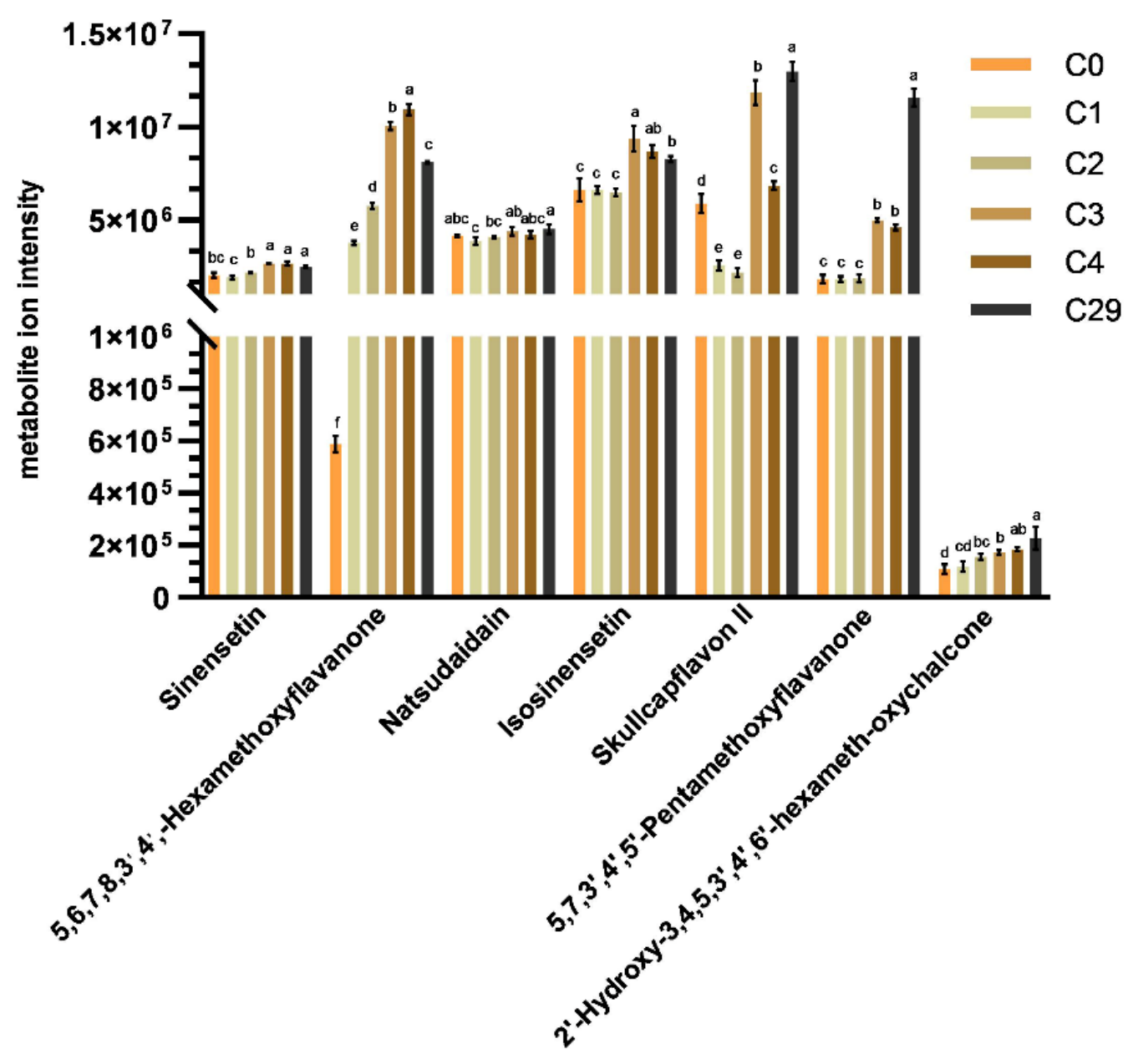

Figure 9

The change of contents of seven polymethoxyflavones 

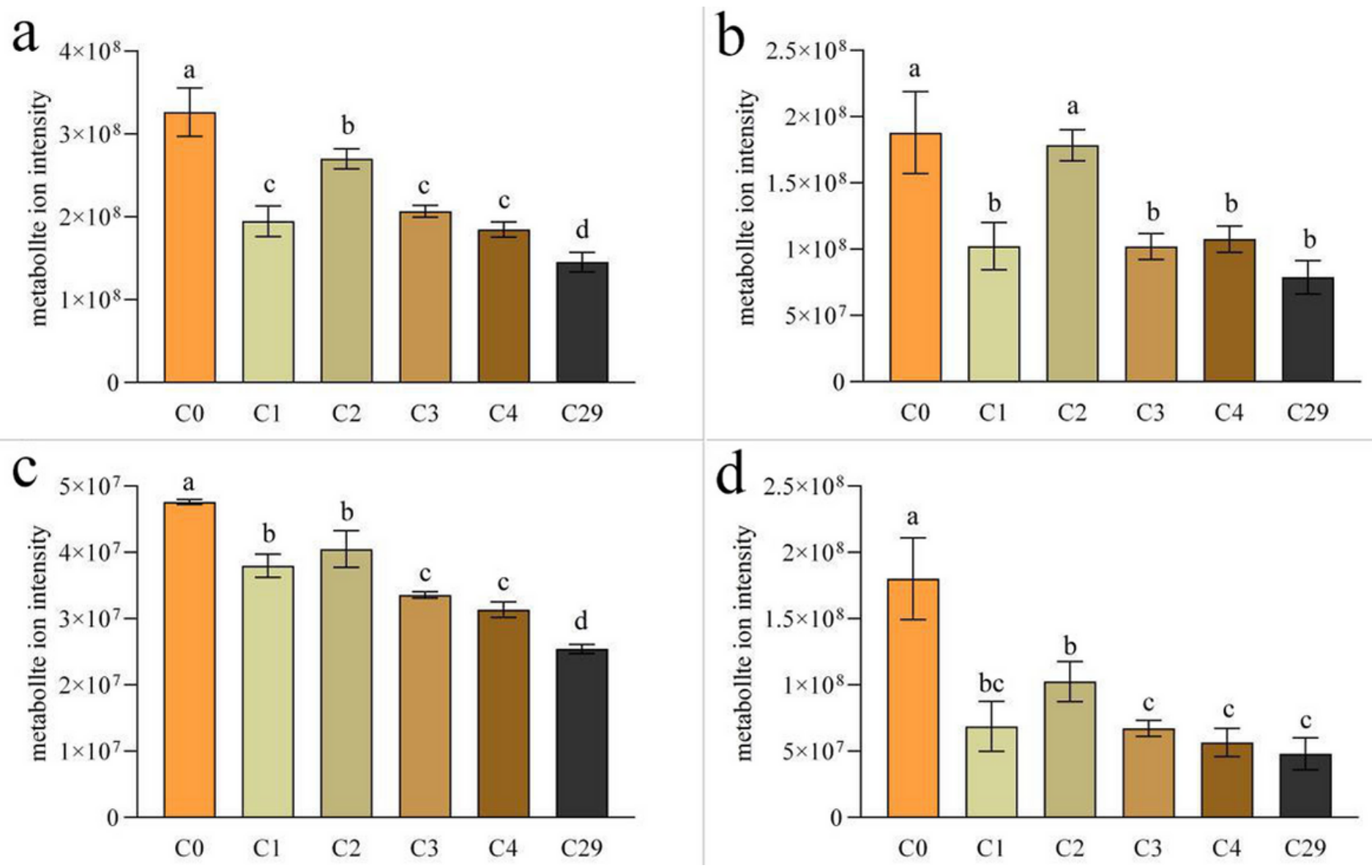

\section{Figure 10}

the total content of flavonoids with lower binding energy than positive control drug in PCRC with different aging periods (a)the total content of flavonoids with lower binding energy than positive control drug at 3CLpro, (b)the total content of flavonoids with lower binding energy than positive control drug at RdRp, (c)the total content of flavonoids with lower binding energy than positive control drug at PLpro, (d)the total content of flavonoids with lower binding energy than positive control drug at Spike.

\section{Supplementary Files}

This is a list of supplementary files associated with this preprint. Click to download.

- renamede24fd.docx 